\title{
Adipose Mesenchymal Stem Cell-Derived Exosomes Relieve Postoperative Abdominal Adhesion By Activating the MAPK-ERK 1/2 and PI3K-Akt Pathways
}

\section{Manyu Shi}

Second Affiliated Hospital of Harbin Medical University

Hengchen Liu

Second Affiliated Hospital of Harbin Medical University

Tingting Zhang

Second Affiliated Hospital of Harbin Medical University

Mingzhao Zhang

Second Affiliated Hospital of Harbin Medical University

\section{Xin Tang}

Second Affiliated Hospital of Harbin Medical University

\section{Zenan Zhang}

Second Affiliated Hospital of Harbin Medical University

\section{Shulong Yang}

Second Affiliated Hospital of Harbin Medical University

Wenjun Lu

Second Affiliated Hospital of Harbin Medical University

\section{Zhitao Jiang}

Second Affiliated Hospital of Harbin Medical University

\section{Qingbo Cui}

Second Affiliated Hospital of Harbin Medical University

Zhaozhu Li ( $\square$ zhaozhu247@163.com )

Second Affiliated Hospital of Harbin Medical University https://orcid.org/0000-0002-8953-8186

\section{Research}

Keywords: Exosomes, Adipose-derived mesenchymal stromal cells, Peritoneal mesothelial cells, Postoperative adhesions, MAPK-ERK1/2, PI3K-Akt

Posted Date: October 5th, 2021 
DOl: https://doi.org/10.21203/rs.3.rs-951403/v1

License: (c) (1) This work is licensed under a Creative Commons Attribution 4.0 International License. Read Full License 


\section{Abstract}

Background: Mesenchymal stem cells (MSCs) have shown potential for peritoneal repair and regeneration. However, the roles of exosomes (Exos) derived from MSCs are not well understood. Therefore, our aim was to elucidate the therapeutic effect of MSC-derived Exos on peritoneal injury.

Methods: Adipose-derived mesenchymal stem cells-derived Exos (ADSC-Exos) were isolated using ultracentrifugation and then injected through the tail vein. Rat peritoneal mesothelial cells (RPMCs) were extracted using enzymatic digestion of the peritoneal cavity. Postsurgical adhesions were brought on by scratching rat cecums. The anti-adhesion effects of ADSC-Exos in rats were evaluated using the Nair's scoring system at 8 and 15 days after abdominal surgery. In addition, the levels of tissue plasminogen activator (t-PA) and plasminogen activator inhibitor-1 (PAl-1) in rat peritoneal fluid were determined via enzyme-linked immunosorbent (ELISA) assays at 8 days after operation. Expression differences in inflammatory and apoptotic markers were detected using immunofluorescence at 4 days after surgery. H\&E staining and immunohistochemistry were employed to observe histological changes and expression changes of extracellular matrix (ECM)-related indices after 15 days. In vitro, ADSC-Exo-driven RPMC proliferation was assessed via 5-ethynyl-2-deoxyuridine assay. Cell migration was determined using scratch-wound and transwell assays. Related signaling networks were estimated based on sequencing and bioinformatics analyses. The roles of the MAPK-ERK1/2 and PI3K-Akt signaling networks were analyzed using immunoblotting.

Results: ADSC-Exos were incorporated into RPMCs, where they induced cell proliferation and migration in proportion to dosage. This was likely mediated via stimulation of the MAPK-ERK 1/2 and PI3K-Akt axes. In vivo, the ADSC-Exos group had a lower adhesion score than the control group at 15 days. At postoperative day 4, inflammation and apoptosis were markedly inhibited by ADSC-Exos. At 8 days, ADSC-Exos treatment decreased PAI-1 expression and increased tPA expression in peritoneal fluid. At 15 days, ADSC-Exos potentially helped regulate the ECM balance.

Conclusions: ADSC-Exos may promote the healing of injured peritoneal tissue, suggesting a promising therapeutic approach for peritoneal adhesions.

\section{Background}

Postoperative adhesions (PAs) are the most frequent and severe complications after almost any type of abdominal or pelvic surgery. Up to $90 \%$ of patients develop PAs after surgical procedures [1-3]. Severe PAs can cause serious complications like intestinal obstruction, persistent abdominal pain, and even female sterility $[4,5]$. Peritoneal mesothelial cells (RPMCs) are metabolically active and play an important role in maintaining serosal homeostasis. A recent report suggested that the mesothelial smooth surface protects against adhesion formation [6]. Peritoneal mesothelial repair differs from the repair of other epithelioid surfaces in that the repair is spread over the injured surface, whereas epithelial healing occurs only at the wound margin. Additionally, the integrity of mesothelial cells can recover relatively quickly, 
regardless of the size of the wound. These observations suggest the existence of a unique healing mechanism, where cells not only migrate from the wound edge to the wound surface, but also separate from opposite surfaces and distant locations to remain on the wound surface. The mitosis rate of mesothelial cells at the edge of wound increased from $0.16-0.5 \%$ to $28-60 \%$ at $24-48 \mathrm{~h}$ post-injury [7, 8]. Recent data have shown that the recovery of surgically injured mesothelial tissue reduces peritoneal adhesion and fibrosis, thereby improving the repair and function of the peritoneal structure $[9,10]$. Therefore, promoting the recovery of peritoneal mesothelial cells may serve as a new therapeutic strategy for reducing PAs.

Mesenchymal stem cells are a subset of adult stromal cells with multidirectional differentiation potential, which have demonstrated great potential in preclinical studies [11]. Adipose-derived mesenchymal stem cells (ADSCs) are abundantly available and convenient to obtain, which makes them more conducive for clinical applications. Moreover, ADSCs are precursor cells that exist in adipose tissue and that have received increasing attention recently. Because of its self-renewal and differentiation potetial, ADSCs are commonly used in tissue repair and injury healing. Mounting evidence revealed that ADSCs administration can significantly enhance repair and regeneration of the peritoneum [12-15].

Exosomes (Exos) are small membranous vesicles that contain a variety of proteins, DNA, mRNAs, and microRNAs (miRNAs), which are important mediators of intercellular communication and serve essential roles in immune regulation, cell migration, and tissue regeneration $[16,17]$. Recent findings indicate that Exos released by mesenchymal stem cells (MSCs) modulate inflammatory responses $[18,19]$ and promote wound healing $[20,21]$. However, it is not clear whether ADSC-derived exosomes (ADSC-Exos) can relieve peritoneal injury.

We speculated that ADSC-Exos relieves acute peritoneal injury by promoting peritoneal injury healing and the extracellular microenvironment. To test this hypothesis, we examined the ADSC-Exos-mediated regulation of (1) inflammation, regeneration, healing, and PA after peritoneal injury in vivo and (2) proliferation and migration of peritoneal mesothelial cells and the related mechanisms in vitro.

\section{Results}

\section{Characterization of ADSCs, ADSC-Exos, and RPMCs}

Flow cytometry was used to detect positive markers CD90 (99.6\%) and CD105 (99.5\%), as well as negative markers CD34 (0.94\%) and CD45 (0.29\%) (Figure 1A). ADSCs showed a typical fusiform morphology and potential for multidirectional differentiation (Figure 1B-E). RPMC showed a typical cobblestone appearance (Figure $1 F$ ), and the immunofluorescence staining results of E-cadherin, vimentin and cytokeratin 19 were positive (Figure 1G-I). TEM analysis revealed that ADSC-Exos had a round vesicle-like structure with a mean diameter of $111.9 \mathrm{~nm}$ (Figure 2A, B). Western blotting indicated the expression of CD9, TSG101, and HSP70 in Exos (Figure 2C). Moreover, ADSC-Exos were incorporated by RPMCs and displayed red fluorescence (Figure 2D). 


\section{Intravenous injection of ADSC-Exos reduced adhesion scores and alleviated inflammation in acute peritoneal adhesions}

Fifteen days after peritoneal scraping, abdominal adhesions were evaluated after laparotomy. The degree of adhesion differed significantly among the groups. Rats treated with PBS (Control group) had dense adhesions and higher average adhesion scores. Adhesions were significantly reduced in rats injected intravenously with ADSC-Exos (Figure $3 A$ ). We observed marked differences in the scores and severity of adhesions between the groups (Figure $3 B$ ). However, at 8 days after peritoneal scraping, no obvious difference was detected between the ADSC-Exos and Control groups. The results are not displayed.

\section{ELISA analysis of t-PA and PAI-1 levels in peritoneal fluid}

The expression of t-PA in the peritoneal fluid of the control group decreased significantly at 8 days after peritoneal scraping, and the expression of PAI-1 increased significantly. However, ADSC-Exos treatment significantly increased t-PA expression and significantly inhibited PAI-1 expression (Figure $3 C, D$ ), relative to controls.

\section{Effects of ADSC-Exo treatment on early inflammatory responses and apoptosis}

We performed H\&E staining to compare the degree of inflammation in the serosal layer on the cecal surface of rats in each group on day 8 . The cecum serosal in the control group was significantly damaged, and plasma cells, granulocytes and macrophages were significantly increased as seen by using H\&E staining (Figure 4A).

Subsequently, we examined the significance of ADSC-Exos in regulating early inflammatory responses in rats. CD163 (an anti-inflammatory M2-type macrophage marker) expression was significantly elevated in the ADSC-Exos cells after 4 days, relative to controls (Figure 4B). By contrast, CCR7 (a pro-inflammatory M1-type macrophage marker) expression was significantly lower (Figure $4 C$ ). Correspondingly, IL-10 (an M2 macrophage-stimulating factor) levels in the ADSC-Exos cells were higher than controls (Figure 4D), whereas the expression of IL-6 (an M1 macrophage-stimulating factor) was significantly lower (Figure $4 E$ ). In addition, we found that Cox-2 (pro-inflammatory factor) expression was drastically lower in the treated groups (Figure 4F).

We then examined the significance of ADSC-Exos on the apoptosis profiles after peritoneal scratch injury. Based on our immunofluorescence data, cleaved caspase-3 (an apoptotic cell marker) levels in intestinal wall cells decreased significantly in the ADSC-Exos groups, relative to the injured controls (Figure 4G). The quantitative analysis results of all the above results are shown in Figure $4 \mathrm{H}$. 


\section{ADSC-Exos improved peritoneal healing and modulated ECM formation}

To evaluate the regeneration ability of damaged peritoneal mesothelial cells in rats. We next, immunohistochemically stained E-cadherin, a mesothelial cell marker, to evaluate the continuity of peritoneal mesothelial layer in damaged rats. In our study, a continuous and intact cell layer was detected on the caecum surface of the sham group. No mesothelial cells were observed on the injured peritoneal surface in the control group. Similarly, the mesothelial cell layer was well repaired in the ADSC-Exos group, and its continuity was similar to that in the sham group. The ADSC-Exos group can significantly promote the repair of injured mesothelial cells on the peritoneal surface (Figure 5A).

We also examined the significance of ADSC-Exos on the peritoneal ECM-related factors Collagen I (COLI), matrix metalloproteinase-9 (MMP-9), and tissue inhibitor of matrix metalloproteinase-1 (TIMP-1). On day 15, relative to controls, TIMP-1 expression was elevated in the ADSC-Exos treatment group (Figure $5 B$ ), and MMP-9 and COLI expression were downregulated (Figure 5C, D). The myofibroblast marker aSMA showed a decreasing trend in the ADSC-Exo-treatment group at 15 days (Figure 5E). The presence of fibronectin was associated with scar formation after peritoneal injury, and fibronectin expression decreased drastically in the ADSC-Exos group (Figure 5F). Quantitative analysis of these results is shown in Figure $5 G$.

\section{Cell proliferation and migration}

Next, we assessed the outcomes of different concentrations of ADSC-Exos and the two pathway inhibitors on proliferation and migration of RPMC. The results of EdU revealed that Exos remarkably accelerated cell proliferation in proportion to dosage (Figure 6A, B). The data of transwell assays showed that the peritoneal mesothelial cell migration gradually increased $24 \mathrm{~h}$ following treatment with varying concentrations of Exos (Figure 6C, D). Scratch tests showed similar results (Figure 6E, F).

\section{miRNA-sequencing and bioinformatics analyses of ADSC- Exos}

To explore whether ADSC-Exos promote RPMC proliferation and migration, we sequenced miRNAs in ADSC-Exos. We selected the top 10 miRNAs (Figure $7 A, B$ ) for functional-enrichment analysis using the $\mathrm{GO}$ and KEGG databases. The most enriched biological process (BP) category was regulation of "cell proliferation" (Figure $7 C$ ). The most common cellular component (CC) category was "membrane" (Figure 7D), and the most enriched molecular function (MF) category was "ATP binding" (Figure 7E). KEGG analyses revealed that "PI3K/Akt" and "MAPK" were the major signal pathways enriched (Figure 7F). Hence, we boldly speculated that ADSC-Exos accelerated RPMC proliferation and migration via the MAPK-ERK 1/2 and PI3K-Akt signaling pathways. 


\section{Mechanism through which ADSC-Exos regulated RPMC proliferation and migration}

Based on the sequencing results obtained with ADSC-Exos, we studied the MAPK-ERK 1/2 and PI3K-Akt cell pathways to explore the mechanism whereby ADSC-Exos regulate the proliferation and migration of RPMCs. Western blotting analysis was performed to assess the activation of these two pathways. Based on our analysis, ADSC-Exos markedly increased p-ERK1/2 and p-Akt protein levels in RPMCs in proportion to dosage (Figure $8 A-C$ ). These findings suggest that ADSC-Exo-dependent proliferation and migration may be mediated by MAPK-ERK $1 / 2$ and PI3K-Akt signaling. To further investigate the regulatory effects of these two networks on RPMCs, we pretreated them with the signaling inhibitors LY294002 and PD98059 prior to ADSC-Exos exposure. Based on our data, these signaling inhibitors significantly inhibited ADSC-Exo-induced phosphorylation of Akt and ERK1/2 (Figure 8D-G). In functional terms, the results of the EdU experiments showed that ADSC-Exos significantly weakened the effect of inhibiting MAPK-ERK 1/2 and PI3K-Akt signals on RPMC proliferation (Figure $8 \mathrm{H}$,I). Similarly, ADSC-Exos promoted peritoneal mesothelial cell migration, which was also markedly inhibited by both signaling inhibitors (Figure 8J-M).

\section{Discussion}

At present, the main pharmacological measures used to prevent abdominal adhesions include (1) promoting fibrinolysis, (2) inhibiting fibroblast proliferation, (3) reducing the initial inflammatory response and exudation, (4) using a physical barrier to isolate the fibrin coating on the damaged surface, and (5) suppressing exudate agglutination. However, other investigators have reported that these treatments are not significantly effective [22]. The reason why these approaches are not effective in preventing adhesion may be that the functions of mesothelial cells on the peritoneal surface were neglected.

Mesothelial cells are the main constituents of the peritoneum. Mesothelial cells serve as a mechanical defensive barrier by covering the abdominal wall and surfaces of internal organs with a single layer of flat epithelial cells to prevent subcutaneous tissue exposure and microbial invasion. During the early stage of abdominal adhesions, if effective procedures are adopted to improve the regeneration and migration capability of mesothelial cells, and complete mesothelial layer is reconstructed, then the formation of mature adhesions can be efficiently suppressed [9, 23, 24]. Moreover, mesothelial cells, which are essential proteases in the fibrinolytic pathway, are the main source of t-PA [25]. Therefore, increasing the number of mesothelial cells and, thus, the level of t-PA is one method to prevent adhesion. In this study, rapid healing of peritoneal mesothelial cells, peritoneal fluid secretion of t-PA increased, and PAI-1 secretion decreased in ADSC-Exo-treated rats. Taken together, these evidences indicate that the rapid enhancement of mesothelial cell regeneration is a potential approach for preventing adhesion formation. Mounting evidence suggests that MSC-derived Exos can suppress the early inflammatory response following peritoneal injury [26] and accelerate tissue healing [27]. Nevertheless, the exact 
mechanism of the ADSC-Exos-mediated regulation of peritoneal injury and mesothelial cell healing remains unclear.

Our work was the first to demonstrate that intravenous administration of ADSC-Exos can promote peritoneal repair after acute peritoneal injury in rats. Proliferation and migration are absolutely essential to the repair of peritoneal injury $[26,28]$. In vitro, we examined the significance of ADSC-Exos on the proliferation and migration of peritoneal mesothelial cells. We foundrevealed that ADSC-Exos promoted the proliferation and migration of peritoneal mesothelial cells in proportion to dosage. Exos from other cell sources may provide comparable results, as multiple findings revealed that Exos originating from MSCs can accelerate cell proliferation and migration, thereby expediting tissue injury repair [29]. Exos mostly function using miRNAs [30]. We sequenced miRNAs in ADSC-Exos to elucidate the mechanism whereby ADSC-Exos promote proliferation and migration of peritoneal mesothelial cells. The top 10 most highly expressed miRNA target genes were predicted and analyzed using bioinformatics. Enrichment analysis revealed that the "PI3K-Akt" and "MAPK" were the most enriched networks. Hence, we hypothesize that ADSC-Exos accelerate the proliferation and migration of peritoneal mesothelial cells by activating the MAPK-ERK $1 / 2$ and PI3K-Akt pathways. Consistent with this hypothesis, ADSC-Exos induced rapid phosphorylation of Akt and ERK, which was abrogated in presence of inhibitors. These data suggest that the pro-survival signals operating in RPMCs can be quickly stimulated by ADSC-Exos, thus improving their survival ability. We also revealed that ADSC-Exo-driven proliferation and migration were downregulated by MAPK-ERK 1/2 and PI3K-Akt pathway inhibitors. In summary, these data suggest the possibility that ADSC-Exos promote RPMC proliferation and migration by triggering activation of the MAPK-ERK 1/2 and PI3K-Akt axes.

We first examined the significance of ADSC-Exos on peritonealinjury and peritoneal adhesion in vivo, and we established a complete rat model of acute peritoneal adhesion. We revealed that ADSC-Exos significantly promoted peritoneal healing and prevented the formation of peritoneal adhesions in rats, relative to the PBS-controls.

The early inflammatory response serves a crucial function in peritoneal injury and directly regulate peritoneal mesothelium healing. Macrophages are the most common cell type in damaged peritoneum tissue, and previous data showed that macrophages were present on day 1 after the induction of adhesion [31]. Macrophage functions primarily depend on the differentiation state and can affect wound healing in multiple organs [32]. M1 macrophages play important roles in ECM degradation during the inflammatory response [33]. However, M2 macrophages participate in wound healing and tissue remodeling via ECM production [34]. In this study, 4 days after peritoneal injury, we found significantly fewer CCR7+ M1 macrophages and significantly more CD163+ M2 cells in the ADSC-Exos cells after peritoneal injury, when compared with the corresponding levels in the other groups. In addition, our results revealed increased levels of the M2-stimulating factor IL-10, elevated inflammatory cytokines expression, and reduced IL-6 expression. Based on these evidences, we suggest that ADSC-Exos can promote antiinflammatory activity and peritoneal injury healing by regulating the differentiation status of macrophages and related cytokines. Next, COX-2 (pro-inflammatory factor) has been strongly associated 
with adhesion and fibrosis after peritoneal injury [35]. In this study, COX-2 expression significantly decreased in the treated rats after peritoneal injury, suggesting that ADSC-Exos are crucial to the repair and anti-inflammatory effects following peritoneal injury.

H\&E staining showed that fewer neutrophils infiltrated the serosal of the colon in the ADSC-Exos group. This finding indicated that ADSC-Exos positively affected the repair of peritoneal injury, which was further confirmed by the changes observed in ECM-related factors during peritoneal repair. Peritoneal adhesions are caused by peritoneal injury, infection, ischemia, or oxidative stress. These stimuli can cause peritoneal mesoderm cell dysfunction, resulting in inflammatory exudation rich in fibrinogen, which is then degraded primarily by fibrinolysis. Adhesions form when fibrinolysis of the damaged peritoneum is insufficient due to excessive deposition of ECM components. Upregulation of fibrinolytic activity leads to decreased ECM protein expression, and COLI is the most important component of the ECM [36]. The injury-control group showed much higher expression of COLI than the sham-operation group, indicating that excess ECM proteins were deposited, but could be reduced by administering ADSC-Exos. MMPs comprise a family of proteases whose main activity is degradation of the ECM. MMP-3, an important member of the MMP family, can inhibit PAI-1 expression and elevate plasminogen levels. In turn, plasminase activates ProMMP-9 to form MMP-9, which assists in remodeling the ECM [37]. TIMPs are natural inhibitors of the MMP family members, which inhibit MMPs by degrading the ECM. Balanced expression between MMPs and TIMPs has been reported to play essential roles in ECM remodeling [38, 39]. Here, we demonstrated that TIMP-1 expression was upregulated whereas MMP- 9 expression was downregulated at 15 days after peritoneal injury in the ADSC-Exo-treated group. Based on our data, ADSCExos exposure balances peritoneal ECM degradation and synthesis by regulating the metabolic balance between TIMP-1 and MMP-9. In addition, the levels of a-SMA and fibronectin were also detected, which displayed a decreasing trend. Previous evidences showed that over-expression of a-SMA and fibronectin inhibitors prevented postoperative scaring and fibrosis development after PAs [40, 41]. In conclusion, our findings indicate that ADSC-Exos critically suppresses development of peritoneal adhesions and accelerates the rapid healing of mesenchymal cells after peritoneal injury.

However, this study had some limitations. First, we did not investigate increased Akt and ERK phosphorylation in RPMCs after ADSC-Exos stimulation at several time points (e.g., 15, 45, and 60 min), and the time point used (30 min) may be suboptimal. In addition, we selected tail vein injection as the method of administration. Due to the limitation of experimental conditions, we did not verify that the Exos were distributed throughout the body after tail vein injection. Third, although we estimated and verified the network whereby ADSC-Exos exerts functional roles through sequencing, the target miRNAs involved require further exploration.

\section{Conclusions}

Our work suggests that the intravenous ADSC-Exos injection can significantly inhibit adhesion formation after peritoneal injury via suppressing inflammation, reducing apoptosis, and enhancing the fibrinolytic systems. Nevertheless, ADSC-Exos can significantly promote the proliferation and migration of RPMC in a 
dosage-proportional manner, which may be reliant on the stimulation of the MAPK-ERK 1/2 and PI3K-Akt signaling pathways. These findings support the clinical use of ADSC-Exos to manage peritoneal injuries.

\section{Methods}

\section{Animals}

Male Sprague-Dawley (SD) rats were obtained from the Animal Experimental Center of Harbin Medical University (Harbin, Heilongjiang, China). All animal protocols strictly followed the guidelines of the Care and Use of Laboratory Animals of the United States National Institutes of Health, and our work received ethical approval from the appropriate committee (approval number Ky2018-135). Rats were randomly placed into one of three groups (6 rats/group), and were euthanized at 4, 8, and 15 days post surgery for the corresponding experiments. All the rats were housed in cages with constant humidity (60 $\pm 5 \%)$ and temperature $\left(23 \pm 2^{\circ} \mathrm{C}\right)$ with a $12-\mathrm{h} / 12$-h light/dark cycle and free access to laboratory feed and water.

\section{ADSCs and RPMCs harvest and identification}

ADSCs were extracted as reporter earlier [12]. Male SD rats, with body weight between 100-150 g, were euthanized via $2 \%$ sodium pentobarbital $(40 \mathrm{mg} / \mathrm{kg})$ intraperitoneal injection. Following a $75 \%$ alcoholmediated disinfection, the skin was incised diagonally at the groin to remove subcutaneous fat. The adipose tissue was rinsed thrice with sterile cold phosphate-buffered saline (PBS) and chopped. The extracellular matrix (ECM) was lysed in 0.2\% COLI (Sigma-Aldrich, St. Louis, MO, USA) for 1 hour at $37^{\circ} \mathrm{C}$, before undergoing centrifugation at $1000 \times \mathrm{g}$ for $10 \mathrm{~min}$. ADSCs were cultured in DMEM/F12 (Invitrogen, Carlsbad, CA, USA) with 10\% fetal bovine serum (FBS; Biological Industries, Kibbutz Beit-Haemek, Israel) and $1 \%$ penicillin-streptomycin (Beyotime, Shanghai, China), and incubated at $37^{\circ} \mathrm{C}$ and $5 \% \mathrm{CO}_{2}$. Following a $48 \mathrm{~h}$ incubation, the medium was changed twice a week, with care being taken to not remove the adherent cells. Phenotypic analysis was performed at passage 3 to begin generating ADSCs. Flow cytometry was employed to detect levels of different surface markers. The ADSCs were cultured separately in adipogenic, osteogenic, or chondrogenic differentiation medium (Cyagen, Santa Clara, CA, USA) to identify their differentiation potentials.

RPMCs were isolated as previously described [42]. Briefly, $25 \mathrm{ml}$ of $0.25 \%$ trypsin and $0.02 \%$ EDTA-Na2 were injected into the intraperitoneal region of rats. Peritoneal effusions were collected $2 \mathrm{~h}$ later under aseptic conditions. The isolated RPMCs were grown in DMEM/F12 medium with $20 \% \mathrm{FBS}$ at $37^{\circ} \mathrm{C}$ and $5 \% \mathrm{CO}_{2}$. RPMCs were verified by certain characteristics, such as, a polygonal pebble morphology and presence of mesothelium-specific markers cytokeratin-19, vimentin, and E-cadherin. Cells between passages 2 and 3, grown as a monolayer to $80 \%$ confluence, were employed in subsequent examinations.

\section{Isolation, identification, and analysis of ADSC-Exos}


Once the ADSCs achieved $80 \%$ confluency, the old culture medium was removed and Exo-depleted medium was introduced. After $24 \mathrm{~h}$, the culture medium was collected without ADSCs before sequential centrifugation at $300 \times \mathrm{g}$ for $10 \mathrm{~min}, 3000 \times \mathrm{g}$ for $10 \mathrm{~min}, 10,000 \times \mathrm{g}$ for $30 \mathrm{~min}$, and $100,000 \times \mathrm{g}$ for $2 \mathrm{~h}$ for Exos extraction. The Exos were mixed in PBS and total protein concentrations in the Exo samples were determined via a bicinchoninic acid assay (Beyotime). Nanoparticle tracking analysis (NTA), transmission electron microscopy (TEM), and western blotting were then employed to characterize the isolated Exos. The abovementioned experiments were repeated thrice.

\section{Cellular internalization of ADSC-Exos}

ADSC-Exos were exposed to $1 \mu \mathrm{M}$ PKH26 (Sigma-Aldrich) for $5 \mathrm{~min}$, and ultracentrifugation was performed to remove excess dye. The labeled Exos were added into serum-free RPMC culture medium and maintained overnight in an incubator. Hoechst 33342 dye (UE, China, Suzhou) was used to stain the nuclei and subsequently, images were captured with a fluorescence microscope (Leica, Wetzlar, Germany).

\section{MiRNAs high-throughput sequence and data analyses}

The miRNAs in ADSC-Exos were sequenced at OE Biotech Company (Shanghai, China). In short, ADSCExos miRNAs were isolation with the RNA isolation Kit (Takara), and the extraction quality was checked with an Agilent 2100 Bioanalyzer (Agilent Technologies). Next, $20 \mathrm{ng}$ of Exo RNA was isolated for library construction. Finally, the libraries were sequenced with an Illumina HiSeq 2500 instrument (Illumina, San Diego, CA, USA). The top 10 miRNAs exhibiting the highest expression in ADSC-Exos were studied using the miRanda software to predict target genes. DAVID (https://david.ncifcrf.gov/) and KOBAS 3.0 (http://kobas.cbi.pku.edu.cn/kobas3/) were employed for Gene Ontology (GO) and Kyoto Encyclopedia of Genes and Genomes (KEGG) pathway-enrichment analyses for the target genes.

\section{Experimental design and surgical procedures}

All animals were operated upon as described previously [43]. The rats were fasted for $8 \mathrm{~h}$ before the surgery. Male SD rats, with body weight ranging from 200-250 g, were anesthetized via intraperitoneal injection with $2 \%$ sodium pentobarbital $(40 \mathrm{mg} / \mathrm{kg})$. After disinfection with $75 \%$ alcohol, a median abdominal incision of approximately $2.0 \mathrm{~cm}$ was made on the skin. The cecum was extracted and rubbed gently with two pieces of dry gauze on the ventral and dorsal sides, until it lost luster and bleeding spots were visible. The cecum was then put back in place, and the layers were closed. Rats $(n=54)$ were arbitrarily separated into the following three groups: (1) Sham: only the abdominal incision was performed, which was stratified and closed after 3 min of exposure; (2) Control: $100 \mu \mathrm{L}$ PBS was injected through the tail vein for three consecutive days after abdominal closure; (3) ADSC-Exos: $400 \mu \mathrm{g}$ ADSCExos was dissolved in $100 \mu \mathrm{L}$ PBS and administered via the tail vein for three consecutive days after 
abdominal closure. After 4, 8, or 15 days, animals from each group $(n=6)$ were euthanized, and adhesion was evaluated in all animals using a U-shaped incision. After adhesion grading was performed, the tissues and peritoneal fluid were collected for subsequent experiments.

\section{Peritoneal adhesion scoring system}

After euthanasia at 8 and 15 days after peritoneal scraping, the degree of postoperative intraperitoneal adhesion was assessed using the adhesion scoring criteria proposed by Nair et al. [44]. Nair's score (Table 1) was used for evaluating the degree of postoperative intraperitoneal adhesion. The grade evaluators were third party contractors who were blinded to the research design.

\section{Enzyme-linked immunosorbent assay (ELISA) for tissue plasminogen activator (t-PA) and plasminogen activator inhibitor-1 (PAI-1) levels in peritoneal fluid}

Briefly, peritoneal fluids were collected from the abdominal cavities of rats 8 days after peritoneal scraping. The contents of liquid-related factors, t-PA, and PAI- 1 in peritoneal fluid were determined using t-PA and PAI-1 ELISA kits (Shanghai Jianglai Biotechnology Inc., Shanghai, China), following kit operational guidelines.

\section{Histological analysis of peritoneal adhesions}

Rats were sacrificed at 8 and 15 days after scraping ( $n=6$ at each time point/group). When fibrous bands did not form, the cecal wall was taken as the specimen; otherwise, the entire fibrous zone was taken. The specimens were immersed in formalin for $24 \mathrm{~h}$, embedded in paraffin, and sliced into $4-\mu \mathrm{m}$ thick sections. For histology, all specimens received hematoxylin and eosin (H\&E) staining using routine procedures. Observation was done under a light microscope (Nikon Eclipse Ni-U, Tokyo, Japan) at 100x and $200 \times$ magnification. Five randomly selected areas in each section were evaluated by an independent pathologist at $200 \times$ magnification.

\section{Immunohistochemical evaluation of peritoneal adhesions}

For immunohistochemical analyses, the sections were exposed overnight at $4^{\circ} \mathrm{C}$ to antibodies against Ecadherin (rabbit monoclonal; 1:200; ab76319; Abcam), COL I (rabbit monoclonal; 1:200; ab270993; Abcam, Cambridge, UK), MMP-9 (rabbit monoclonal; 1:200; ab76003; Abcam), TIMP-1 (rabbit polyclonal; 1:200; Abcam), a-SMA (mouse monoclonal; 1:200; ab7817; Abcam), or fibronectin (rabbit monoclonal; 1:200; ab199056; Abcam), followed by incubation with secondary horseradish peroxidaseconjugated goat anti-rabbit IgG antibody (1:500; 115-035-003; Jackson ImmunoResearch, Ely, UK).. 


\section{Immunofluorescence staining of peritoneal adhesions}

Next, we stained sections with immunoflourescent markers for inflammation and apoptosis as described earlier [45]. In short, following a blocking step, the sections were exposed overnight at $4^{\circ} \mathrm{C}$ to antibodies against CD163 (rabbit monoclonal; 1:100; ab182422; Abcam), C-C chemokine receptor type 7 (CCR7; rabbit monoclonal; 1:200; ab32527; Abcam), interleukin (IL)-10 (rabbit monoclonal; 1:100; ab33471; Abcam), IL-6 (mouse monoclonal; 1:100; TA500067; Origene), COX-2 (rabbit monoclonal; 1:100; ab179800 Abcam), and cleaved caspase-3 (rabbit monoclonal; 1:100; ab32042; Abcam). Following a PBS-wash, the sections were exposed to a secondary antibody (1:200; SA00003 and SA00009; Proteintech) without light for $1 \mathrm{~h}$, before counterstaining with 4,6-diamidino-2-phenylindole (DAPI, Beyotime) for 10 min. Image capture was done with an Eclipse Ni-U microscope (Nikon) and analysis with the ImageJ software.

\section{Treating RPMCs with ADSC-Exos}

To establish peritoneal injury healing model in vitro, $1 \times 10^{6}$ RPMCs were incubated in 6-well culture plates for $18 \mathrm{~h}$, after which they were randomly divided into four groups. ADSC-Exos were added at concentrations of $0,25,50$, or $100 \mu \mathrm{g} / \mathrm{mL}$ after replacing the RPMC medium with Exo-free medium. Next, to further investigate the associated mechanisms, RPMCs inoculated on a 6-well culture plate were arbitrarily separated into four groups: (1) Control group: the RPMC medium was replaced with Exo-free medium; (2) ADSC-Exos group: the RPMC medium was replaced with Exo-free medium containing 100 $\mu \mathrm{g} / \mathrm{mL}$ ADSC-Exos; (3) ADSC-Exos + LY group: RPMCs were treated with $10 \mathrm{nM}$ LY294002 (a PI3K/Akt inhibitor; MedChemExpress, Monmouth Junction, NJ, USA) for 30 min prior to adding $100 \mu \mathrm{g} / \mathrm{mL}$ ADSCExos; (4) ADSC-Exos + PD group: RPMCs were treated with 50 nM MAPK/ERK1/2 inhibitor PD 98059 (MedChemExpress) for 30 min prior to adding $100 \mu \mathrm{g} / \mathrm{mL}$ ADSC-Exos. The RPMCs in each group were collected after $30 \mathrm{~min}$ or $24 \mathrm{~h}$ later and analyzed using western blotting. In addition, 5-ethynyl-2deoxyuridine (EdU), scratch-wound, and transwell assays were conducted $24 \mathrm{~h}$ later.

\section{Proliferation analysis of RPMC}

RPMC proliferation was assessed with the EdU Assay Kit (UE), following kit operational guidelines. Briefly, RPMCs $\left(1 \times 10^{4} /\right.$ group) were exposed to $50 \mu \mathrm{M}$ EdU for $1 \mathrm{~h}$, then fixed in $4 \%$ paraformaldehyde and stained using the EdU kit. Hoechst dye (UE) for 20 min stained the cell nuclei. At last, quantification of EdU-positive cells was performed manually using a fluorescence microscope.

\section{Migration analysis of RPMC}

To examine cell migratory patterns, we selected scratch-wound and transwell assays. In the scratchwound experiments, ADSCs $\left(1.5 \times 10^{5}\right.$ cells/well) were plated in 6-well plates and allowed to attach 
overnight. A linear wound was generated in the monolayer using a sterile $200-\mu \mathrm{L}$ pipette tip. Serum-free medium containing ADSC-Exos was then introduced to each well. Image capture was done at 0 and $24 \mathrm{~h}$ following ADSC-Exos exposure with the help of an inverted microscope.

For the transwell experiments, RPMCs $\left(1 \times 10^{4} /\right.$ group $)$ were plated in the upper chamber of the transwell plate (Corning Inc., NY, USA). Then, medium with $10 \%$ free-Exos serum and different concentrations of ADSC-Exos was introduced to the lower chamber. Following $24 \mathrm{~h}$, RPMCs on the permeable membrane of the upper chamber underwent fixation in paraformaldehyde, staining with crystal violet. Finally, cells that travelled to the other side of the membrane were counted under a light microscope.

\section{Western blotting analysis}

Briefly, ADSCs lysis was done in RIPA buffer (Beyotime) for protein extraction. The protein concentrations in the lysates were estimated by performing dipicolinic acid assays (Beyotime). Immunoblotting was done with primary rabbit antibodies against TSG101 (monoclonal; 1:2000; ab125011; Abcam), CD9 (monoclonal, 1:2000; ab92726; Abcam), HSP70 (monoclonal; 1:1000; ab2787; Abcam), ERK1/2 (1:1000; Cell Signaling Technology), phospho-ERK1/2 (1:2000; Cell Signaling Technology), Akt (1:1000; Cell Signaling Technology), and phospho-Akt (1:2000; Cell Signaling Technology). A horseradish peroxidaseconjugated goat anti-rabbit antibody (1:5000; Boster, China) was employed as the secondary antibody. The ImageJ software was used for densitometric analysis of the final protein bands.

\section{Statistical analyses}

All data are presented as mean \pm standard deviation. Quantitative data across all groups were compared using one-way analysis of variance and subsequently, the Tukey's test. $P<0.05$ was set as significance threshold.

\section{Abbreviations}

ADSCs, adipose-derived mesenchymal stromal cells; BP, biological process; CC, cellular component; ECM, extracellular matrix; ELISA, enzyme-linked immunosorbent assay; FBS, fetal bovine serum; GO, Gene Ontology; H\&E, hematoxylin and eosin; KEGG, Kyoto Encyclopedia of Genes and Genomes; MF, molecular function; MSC, mesenchymal stem cells; NTA, nanoparticle tracking analysis; PA, postoperative adhesions; PBS, phosphate-buffered saline; RPMC, rat peritoneal mesothelial cells; TEM, transmission electron microscopy

\section{Declarations}

\section{Acknowledgements}


We would like to acknowledge the reviewers for their helpful comments on this paper.

\section{Authors' contributions}

MYS, HCL contributed to cytology experiment, animal experiments; MYS, TTZ contributed to data acquisition, data analysis; MYS, MZZ and XT contributed to manuscript writing; TTZ, SLY, ZNZ and ZTJ provided experimental technical support and final approval of manuscript; QBC and ZZL took part in the experimental design, text revision, and final approval of manuscript.

\section{Funding}

This study was supported by the National Natural Science Foundation of China $(81871837,81572117)$ and the Specialized Research Fund for Doctoral Programs in Colleges and Universities of China (20132307110007).

\section{Availability of data and materials}

The datasets used and/or analyzed during the current study are available from the corresponding author on reasonable request.

\section{Ethics approval and consent to participate}

The experimental protocol about animals was approved by the Harbin Medical University Ethics Committee.

\section{Consent for publication}

Not applicable

\section{Competing interests}

The authors declare that they have no conflicting interests.

\section{References}

1. Hu J, Fan D, Lin X, Wu X, He X, He X, Wu X, Lan P. Safety and Efficacy of Sodium Hyaluronate Gel and Chitosan in Preventing Postoperative Peristomal Adhesions After Defunctioning Enterostomy: A Prospective Randomized Controlled Trials. Medicine (Baltimore). 2015;94(51):e2354.

2. Beyene RT, Kavalukas SL, Barbul A. Intra-abdominal adhesions: Anatomy, physiology, pathophysiology, and treatment. Curr Probl Surg. 2015;52(7):271-319.

3. Ellis H, Moran BJ, Thompson JN, Parker MC, Wilson MS, Menzies D, McGuire A, Lower AM, Hawthorn RJ, O'Brien F, Buchan S, Crowe AM. Adhesion-related hospital readmissions after abdominal and pelvic surgery: a retrospective cohort study. Lancet. 1999;353(9163):1476-80. 
4. Parker MC, Wilson MS, Menzies D, Sunderland G, Thompson JN, Clark DN, Knight AD, Crowe AM; Surgical and Clinical Adhesions Research (SCAR) Group. Colorectal surgery: the risk and burden of adhesion-related complications. Colorectal Dis. 2004;6(6):506-11.

5. Liakakos T, Thomakos N, Fine PM, Dervenis C, Young RL. Peritoneal adhesions: etiology, pathophysiology, and clinical significance. Recent advances in prevention and management. Dig Surg. 2001;18(4):260-73.

6. Inagaki NF, Inagaki FF, Kokudo N, Miyajima A. Use of mouse liver mesothelial cells to prevent postoperative adhesion and promote liver regeneration after hepatectomy. J Hepatol. 2015;62(5):1141-7.

7. Fotev Z, Whitaker D, Papadimitriou JM. Role of macrophages in mesothelial healing. J Pathol. 1987;151(3):209-19.

8. Whitaker D, Papadimitriou J. Mesothelial healing: morphological and kinetic investigations. J Pathol. 1985;145(2):159-75.

9. Guo Q, Li QF, Liu HJ, Li R, Wu CT, Wang LS. Sphingosine kinase 1 gene transfer reduces postoperative peritoneal adhesion in an experimental model. Br J Surg. 2008;95(2):252-8.

10. Guo H, Leung JC, Cheung JS, Chan LY, Wu EX, Lai KN. Non-viral Smad7 gene delivery and attenuation of postoperative peritoneal adhesion in an experimental model. Br J Surg. 2009;96(11):1323-35.

11. Han Y, Li X, Zhang Y, Han Y, Chang F, Ding J. Mesenchymal Stem Cells for Regenerative Medicine. Cells. 2019;8(8):886.

12. Costalonga EC, Fanelli C, Garnica MR, Noronha IL. Adipose-Derived Mesenchymal Stem Cells Modulate Fibrosis and Inflammation in the Peritoneal Fibrosis Model Developed in Uremic Rats. Stem Cells Int. 2020;2020:3768718.

13. Alatab S, Shekarchian S, Najafi I, Moghadasali R, Ahmadbeigi N, Pourmand MR, Bolurieh T, Jaroughi N, Pourmand G, Aghdami N. Systemic Infusion of Autologous Adipose Tissue-Derived Mesenchymal Stem Cells in Peritoneal Dialysis Patients: Feasibility and Safety. Cell J. 2019;20(4):483-95.

14. Yang CY, Chang PY, Chen JY, Wu BS, Yang AH, Lee OK. Adipose-derived mesenchymal stem cells attenuate dialysis-induced peritoneal fibrosis by modulating macrophage polarization via interleukin6. Stem Cell Res Ther. 2021;12(1):193.

15. Wakabayashi K, Hamada C, Kanda R, Nakano T, lo H, Horikoshi S, Tomino Y. Adipose-derived mesenchymal stem cells transplantation facilitate experimental peritoneal fibrosis repair by suppressing epithelial-mesenchymal transition. J Nephrol. 2014;27(5):507-14.

16. Pegtel DM, Gould SJ. Exosomes. Annu Rev Biochem. 2019;88:487-514.

17. Kalluri R, LeBleu VS. The biology, function, and biomedical applications of exosomes. Science. 2020;367(6478):eaau6977.

18. Shao M, Xu Q, Wu Z, Chen Y, Shu Y, Cao X, Chen M, Zhang B, Zhou Y, Yao R, Shi Y, Bu H. Exosomes derived from human umbilical cord mesenchymal stem cells ameliorate IL-6-induced acute liver injury through miR-455-3p. Stem Cell Res Ther. 2020;11(1):37. 
19. Harrell CR, Jovicic N, Djonov V, Arsenijevic N, Volarevic V. Mesenchymal Stem Cell-Derived Exosomes and Other Extracellular Vesicles as New Remedies in the Therapy of Inflammatory Diseases. Cells. 2019;8(12):1605.

20. Ma T, Fu B, Yang X, Xiao Y, Pan M. Adipose mesenchymal stem cell-derived exosomes promote cell proliferation, migration, and inhibit cell apoptosis via Wnt/ $\beta$-catenin signaling in cutaneous wound healing. J Cell Biochem. 2019;120(6):10847-54.

21. Zhang M, Liu H, Cui Q, Han P, Yang S, Shi M, Zhang T, Zhang Z, Li Z. Tendon stem cell-derived exosomes regulate inflammation and promote the high-quality healing of injured tendon. Stem Cell Res Ther. 2020;11(1):402.

22. Fayez JA, Schneider PJ. Prevention of pelvic adhesion formation by different modalities of treatment. Am J Obstet Gynecol. 1987;157(5):1184-8.

23. Kawanishi K, Nitta K. Cell sheet-based tissue engineering for mesothelial cell injury. Contrib Nephrol. 2015;185:66-75.

24. Bertram P, Tietze L, Hoopmann M, Treutner KH, Mittermayer C, Schumpelick V. Intraperitoneal transplantation of isologous mesothelial cells for prevention of adhesions. Eur J Surg. 1999;165(7):705-9.

25. Sitter T, Spannagl M, Schiff H, Held E, van Hinsbergh VW, Kooistra T. Imbalance between intraperitoneal coagulation and fibrinolysis during peritonitis of CAPD patients: the role of mesothelial cells. Nephrol Dial Transplant. 1995;10(5):677-83.

26. Wang N, Li Q, Zhang L, Lin H, Hu J, Li D, Shi S, Cui S, Zhou J, Ji J, Wan J, Cai G, Chen X. Mesenchymal stem cells attenuate peritoneal injury through secretion of TSG-6. PLoS One. 2012;7(8):e43768.

27. Lucas PA. Stem cells for mesothelial repair: an understudied modality. Int J Artif Organs. 2007;30(6):550-6.

28. Djafarzadeh R, Sauter M, Notohamiprodjo S, Noessner E, Goyal P, Siess W, Wörnle M, Ribeiro A, Himmelein S, Sitter T, Nelson PJ. Recombinant GPI-anchored TIMP-1 stimulates growth and migration of peritoneal mesothelial cells. PLoS One. 2012;7(4):e33963.

29. An Y, Lin S, Tan X, Zhu S, Nie F, Zhen Y, Gu L, Zhang C, Wang B, Wei W, Li D, Wu J. Exosomes from adipose-derived stem cells and application to skin wound healing. Cell Prolif. 2021;54(3):e12993.

30. Barile L, Vassalli G. Exosomes: Therapy delivery tools and biomarkers of diseases. Pharmacol Ther. 2017; 174:63-78.

31. Haney AF. Identification of macrophages at the site of peritoneal injury: evidence supporting a direct role for peritoneal macrophages in healing injured peritoneum. Fertil Steril. 2000;73(5):988-95.

32. Sindrilaru A, Scharffetter-Kochanek K. Disclosure of the Culprits: Macrophages-Versatile Regulators of Wound Healing. Adv Wound Care (New Rochelle). 2013;2(7):357-68.

33. Kou PM, Babensee JE. Macrophage and dendritic cell phenotypic diversity in the context of biomaterials. J Biomed Mater Res A. 2011;96(1):239-60. 
34. Brochhausen C, Schmitt VH, Mamilos A, Schmitt C, Planck CN, Rajab TK, Hierlemann H, Kirkpatrick CJ. Expression of CD68 positive macrophages in the use of different barrier materials to prevent peritoneal adhesions-an animal study. J Mater Sci Mater Med. 2017;28(1):15.

35. Katada J, Saito H, Ohashi A. Significance of cyclooxygenase-2 induced via p38 mitogen-activated protein kinase in mechanical stimulus-induced peritoneal adhesion in mice. J Pharmacol Exp Ther. 2005;313(1):286-92.

36. Diamond MP, El-Hammady E, Wang R, Saed G. Metabolic regulation of collagen I in fibroblasts isolated from normal peritoneum and adhesions by dichloroacetic acid. Am J Obstet Gynecol. 2002;187(6):1456-61.

37. Tang J, Xiang Z, Bernards MT, Chen S. Peritoneal adhesions: Occurrence, prevention and experimental models. Acta Biomater. 2020;116:84-104.

38. Nagase H, Visse R, Murphy G. Structure and function of matrix metalloproteinases and TIMPs. Cardiovasc Res. 2006;69(3):562-73.

39. Cabral-Pacheco GA, Garza-Veloz I, Castruita-De la Rosa C, Ramirez-Acuña JM, Perez-Romero BA, Guerrero-Rodriguez JF, Martinez-Avila N, Martinez-Fierro ML. The Roles of Matrix Metalloproteinases and Their Inhibitors in Human Diseases. Int J Mol Sci. 2020;21(24):9739.

40. Wu Y, Wei G, Yu J, Chen Z, Xu Z, Shen R, Liang T, Zheng L, Wang K, Sun X, Li X. Danhong Injection Alleviates Postoperative Intra-abdominal Adhesion in a Rat Model. Oxid Med Cell Longev. 2019;2019:4591384.

41. Saed GM, Diamond MP. Effects of interferon-gamma reverse hypoxia-stimulated extracellular matrix expression in human peritoneal and adhesion fibroblasts. Fertil Steril. 2006;85 Suppl 1:1300-5.

42. Li D, Lu Z, Li X, Xu Z, Jiang J, Zheng Z, Jia J, Lin S, Yan T. Human umbilical cord mesenchymal stem cells facilitate the up-regulation of miR-153-3p, whereby attenuating MGO-induced peritoneal fibrosis in rats. J Cell Mol Med. 2018;22(7):3452-63.

43. Bi J, Zhang S, Du Z, Zhang J, Deng Y, Liu C, Zhang J. Peripheral serotonin regulates postoperative intra-abdominal adhesion formation in mice. Sci Rep. 2017;7(1):10001.

44. Nair SK, Bhat IK, Aurora AL. Role of proteolytic enzyme in the prevention of postoperative intraperitoneal adhesions. Arch Surg. 1974;108(6):849-53.

45. Iwasaki K, Ahmadi AR, Qi L, Chen M, Wang W, Katsumata K, Tsuchida A, Burdick J, Cameron AM, Sun Z. Pharmacological Mobilization and Recruitment of Stem Cells in Rats Stops Abdominal Adhesions After Laparotomy. Sci Rep. 2019;9(1):7149.

\section{Tables}

\section{Table 1. Nair's macroscopic adhesion classification}




\begin{tabular}{|ll|}
\hline Score & Characteristics \\
\hline 1 & Complete absence of adhesion \\
\hline 2 & Tingle band of adhesion, between viscera or from viscera to abdominal wall \\
\hline 3 & $\begin{array}{l}\text { More than two bands, between viscera or viscera to abdominal wall or whole intestines } \\
\text { forming a mass without being adherent to abdominal wall }\end{array}$ \\
\hline 4 & $\begin{array}{l}\text { Viscera directly adherent to abdominal wall, irrespective of number and extent of adhesive } \\
\text { bands }\end{array}$ \\
\hline
\end{tabular}

\section{Figures}
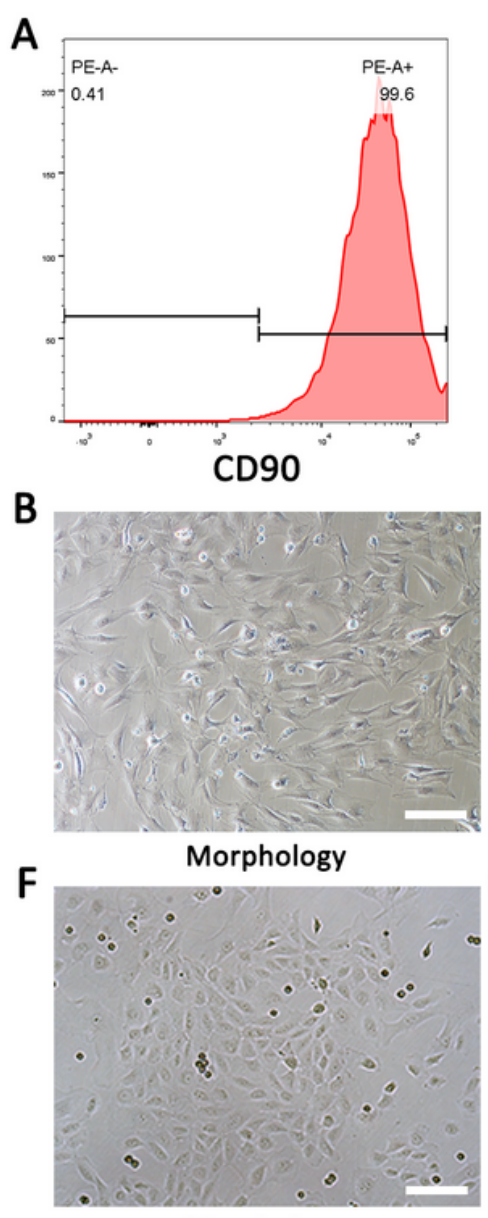

Morphology
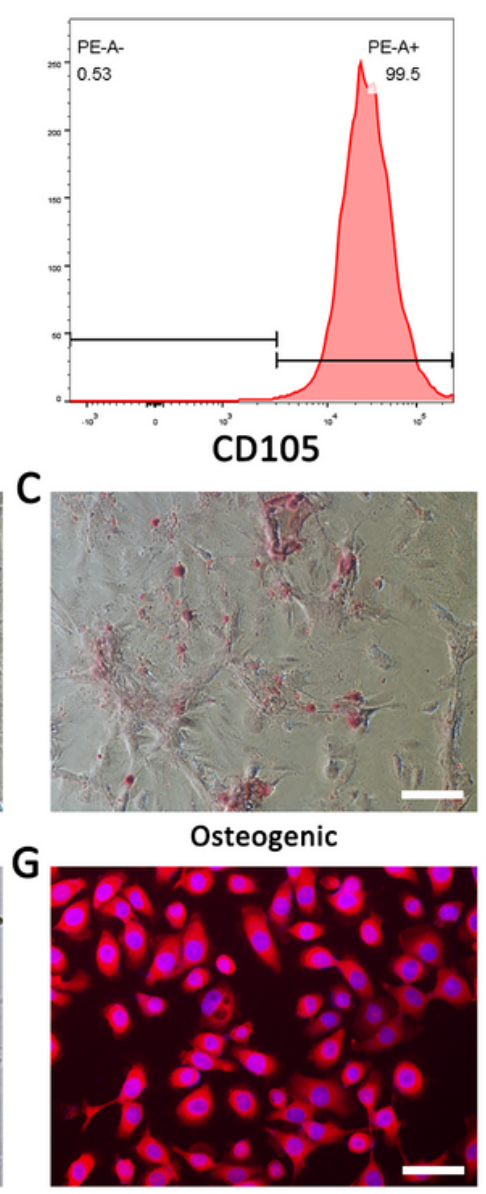

E-cadherin
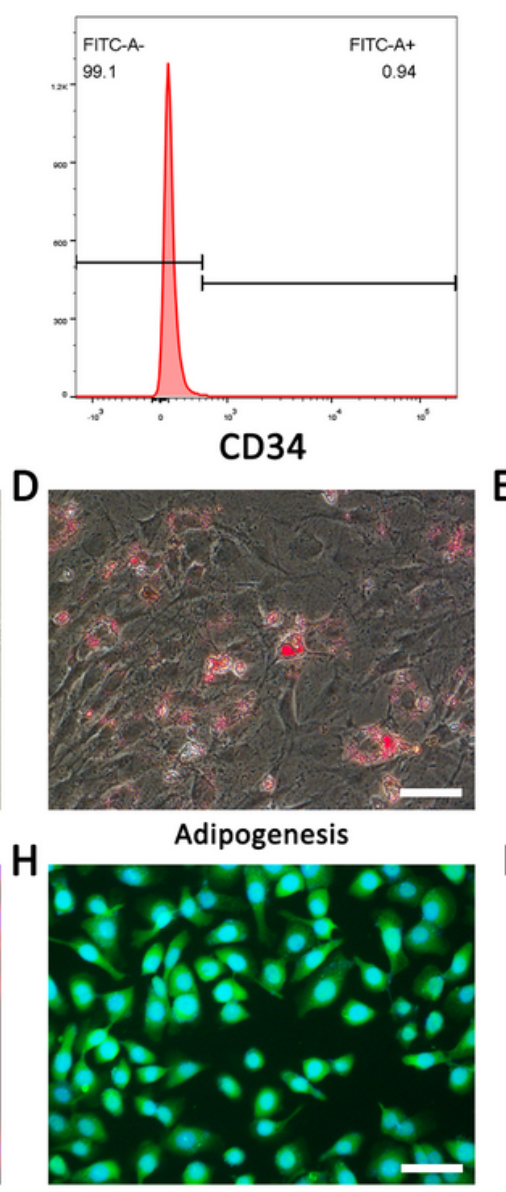

Vimentin

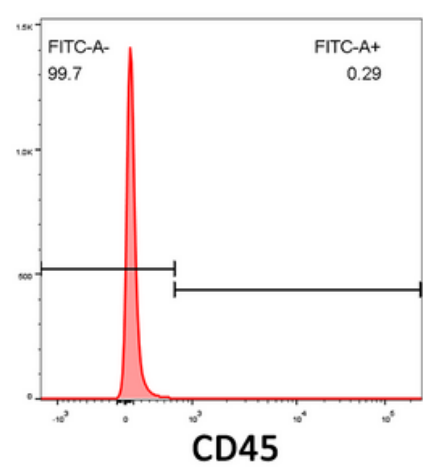

$\mathrm{E}$
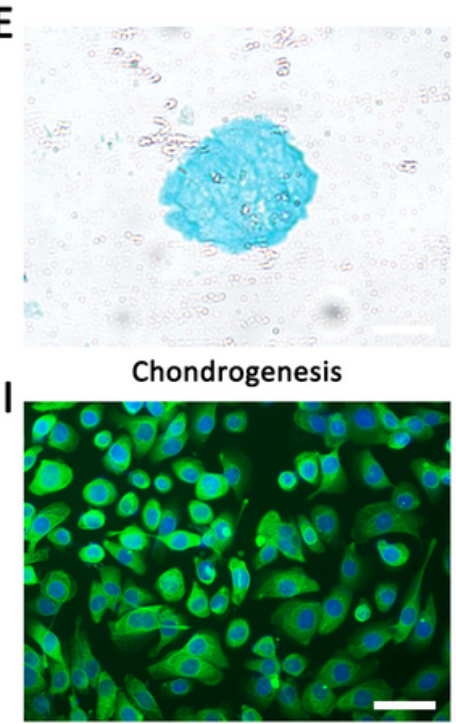

Cytokeratin-19

\section{Figure 1}

Characterization of ADSCs and RPCMs. A Flow cytometry for detection of ADSCs surface markers, including CD90, CD105, CD34, CD45. B Morphology of ADSCs. C-E Osteogenic, adipogenic, chondrogenesis differentiation potential of ADSCs. F Phenotype of rat peritoneal mesothelial cells 
(RPMCs) in culture. G-I Immunofluorescent staining of RPMC (E-cadherin, Vimentin, Cytokeratin-19). Bars, $100 \mu \mathrm{m}$

A

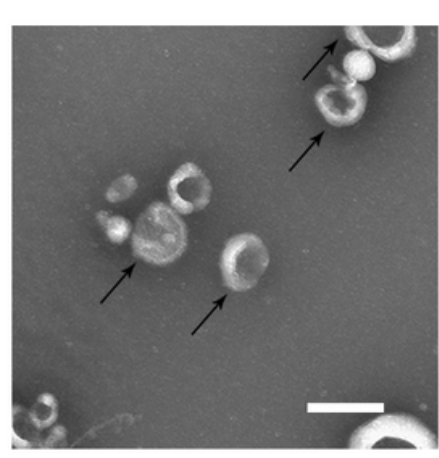

C

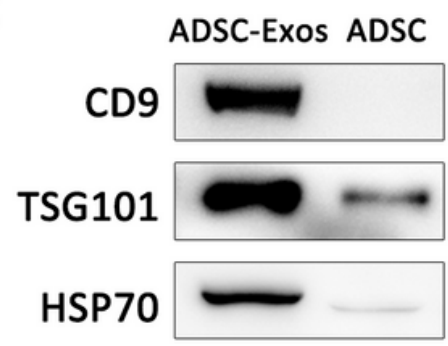

D

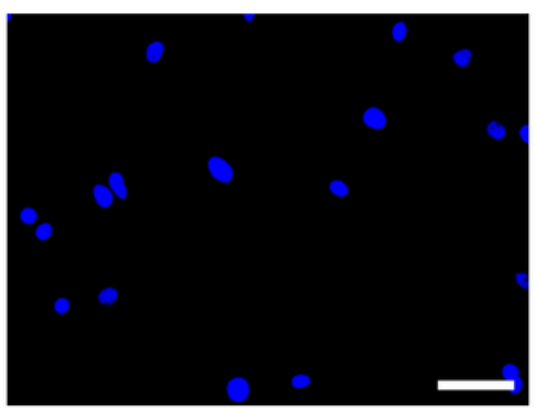

B
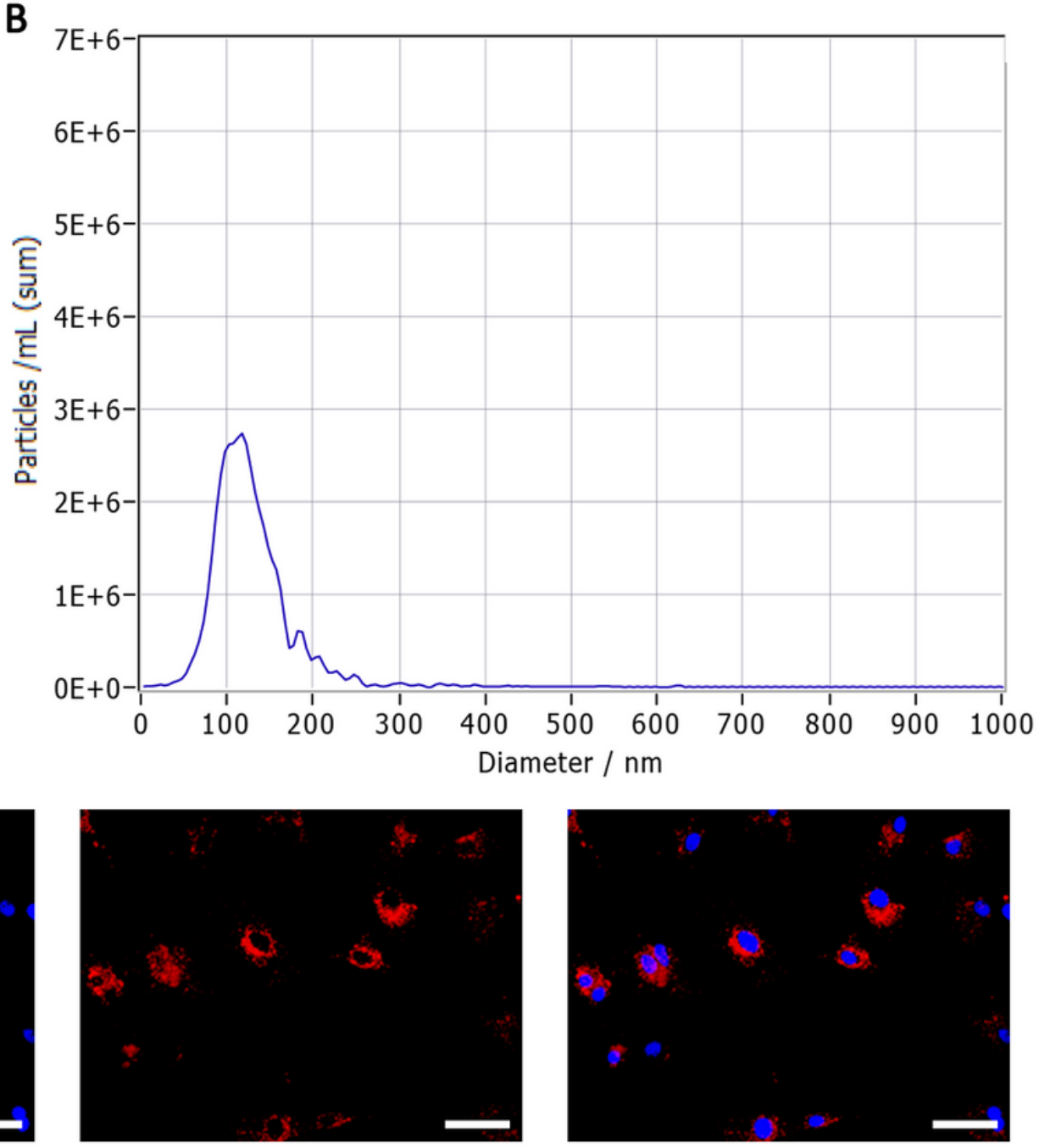

\section{Figure 2}

Characterization of ADSC-Exos. A Morphology observed under transmission electron microscope. B Particle size distribution. C Western blot was used to detect exosomes surface markers. D Fluorescent microscopy analysis of PKH26-labeled ADSC-Exos internalization RPMCs. Nuclei were counterstained with DAPI. Bars (ADSC-Exos), 100 nm; Bars (RPCMs), $100 \mu \mathrm{m}$ 


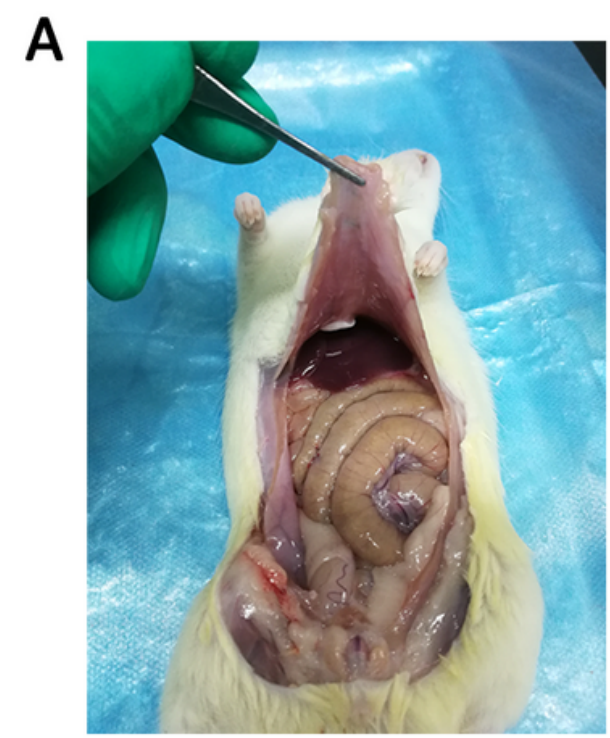

Sham

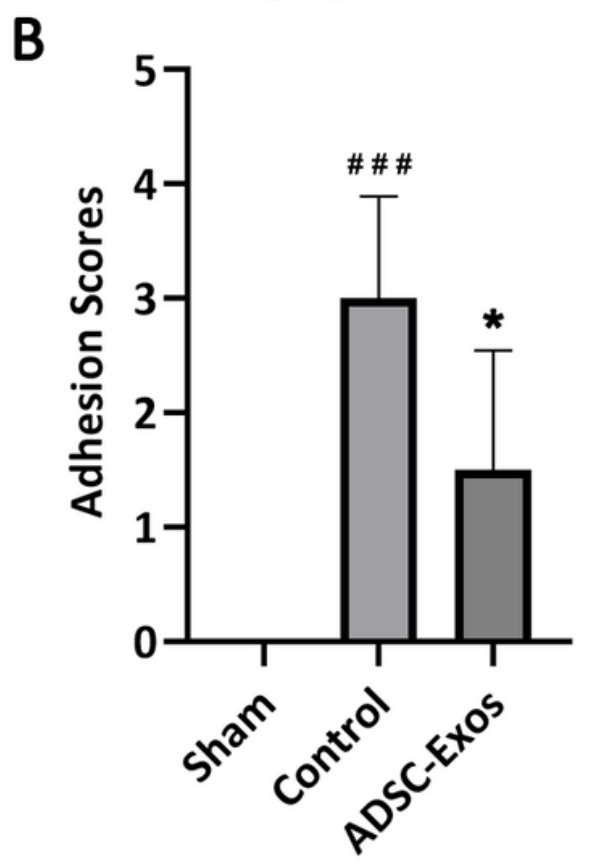

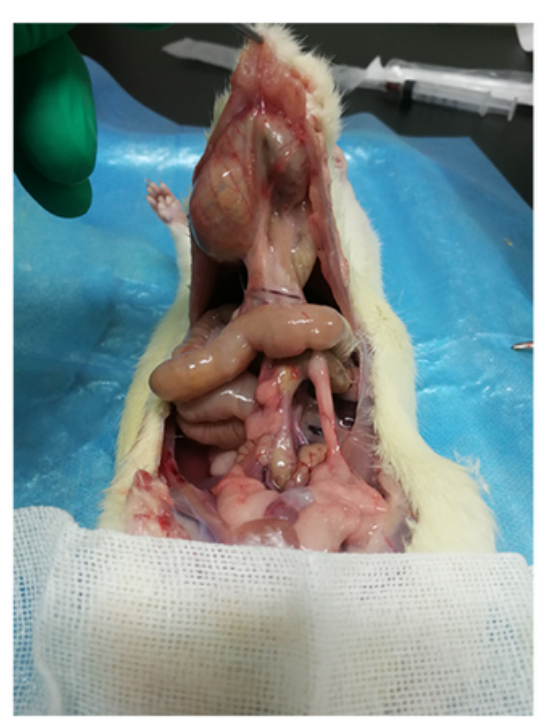

Control

C

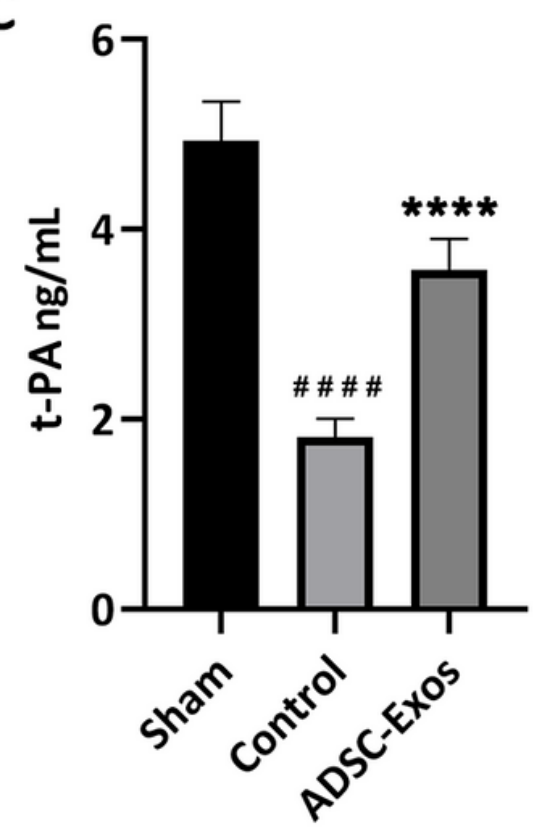

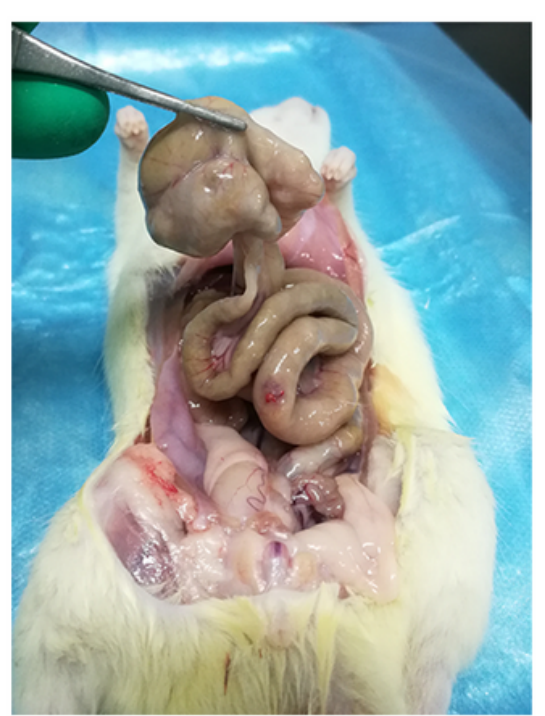

ADSC-Exos

D

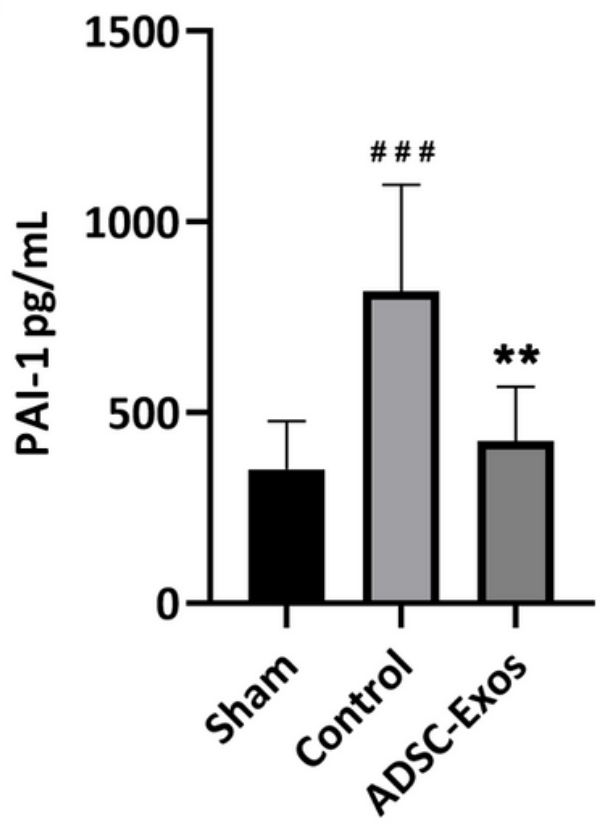

Figure 3

The Nair scores and fibrinolytic system for each group. A The severity of adhesions in each group. B The Nair score for each group. C, D The t-PA and PAI-1 protein levels in peritoneal fluid. Data are represented

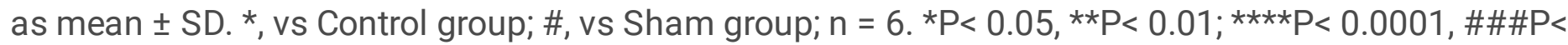
$0.001 ; \# \# \# \mathrm{P}<0.0001$ 
A

HE
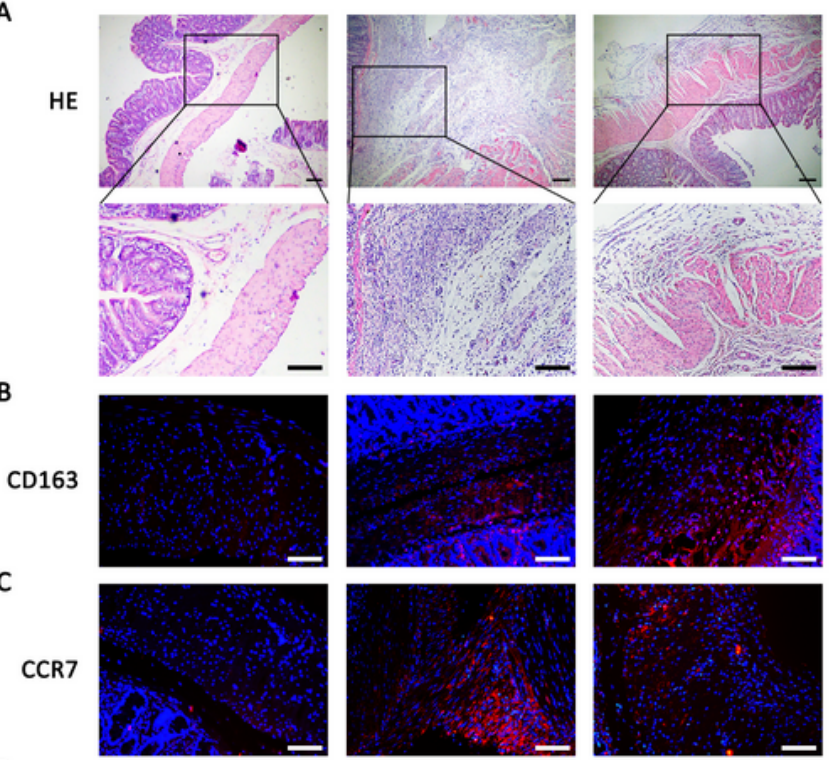

D
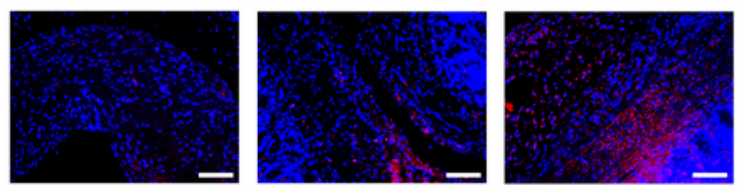

E

IL-6
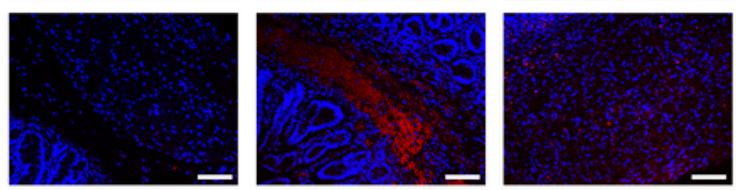

$\mathrm{F}$
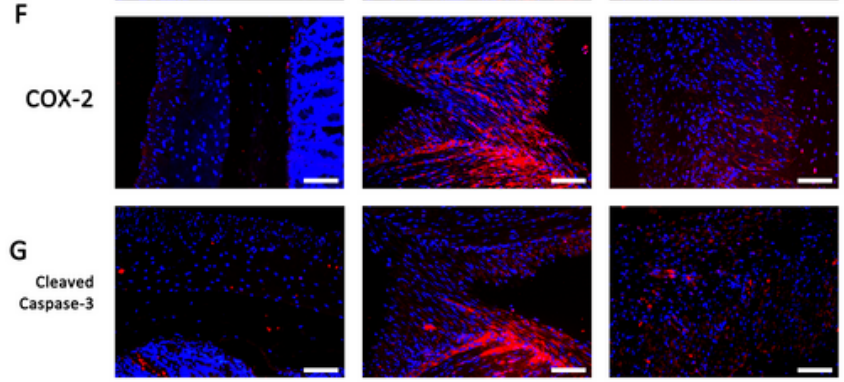

Control

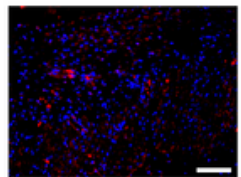

ADSC-Exos

H

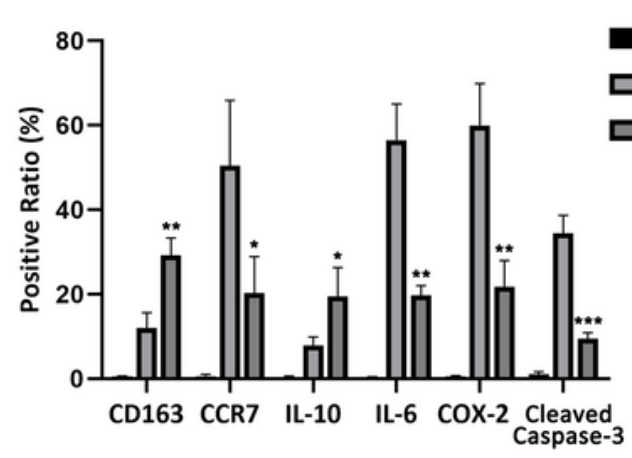

\section{Figure 4}

Impact of ADSC-Exos on peritoneal inflammation and apoptosis. A Hematoxylin-eosin staining of injured tissues (Black tetragonum represents the accumulation of plasma cells, granulocytes and macrophages) B-F The expression of CD163+, CCR7+, IL-10+, IL-6+ and COX-2+ evaluated at 4 days post-surgery by immunofluorescence assay. $G$ Cellular expression of cleaved caspase $3+$ at 4 days after surgery evaluated by immunofluorescence assay. H Positive cell ratio of inflammation-related factors and 
cleaved caspase $3+$ cells at 4 days. Bars, $50 \mu \mathrm{m}$. Data are represented as mean \pm SD. ${ }^{*}$, vs Control group; $\mathrm{n}=6 .{ }^{*} \mathrm{P} \bigotimes 0.05, * * \mathrm{P}<0.01,{ }^{* * *} \mathrm{P}<0.001$

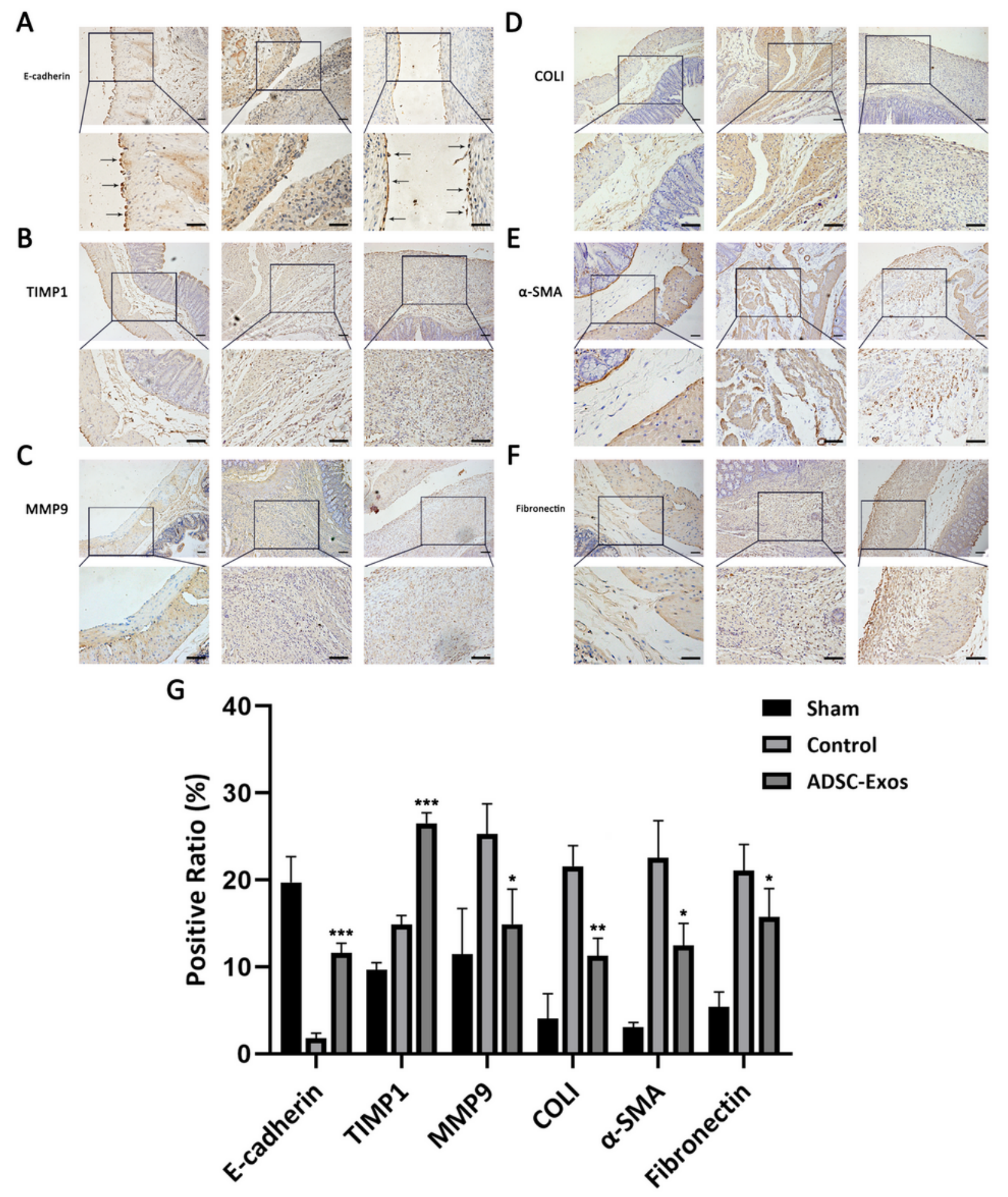

Figure 5

Impact of ADSC-Exos on peritoneal mesothelium cells and peritoneal matrix-related factors. A Immunohistochemical staining of E-cadherin in the injured areas peritoneum in each group of rats. RPMC are shown by black arrrow. B-F The Expression of TIMP-1, MMP-9, COLI, a-SMA, and fibronectin evaluated 
at 15 days post-surgery by immunohistochemistry assay. G Quantitative analysis of peritoneal matrixrelated factors at 15 days. Bars, $50 \mu \mathrm{m}$. Data are represented as mean $\pm \mathrm{SD}$. *, vs Control group; $\mathrm{n}=6 .{ }^{*} \mathrm{P}<$ $0.05, * * P<0.01, * \star * P<0.001$

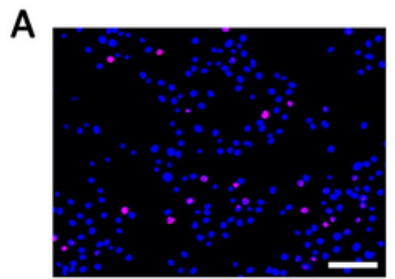

C

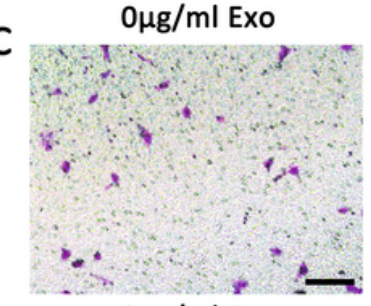

E
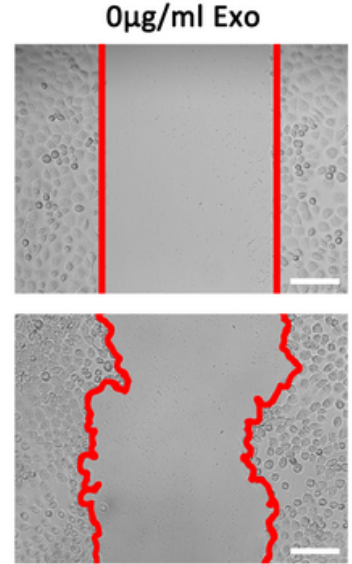

$0 \mu \mathrm{g} / \mathrm{ml}$ Exo

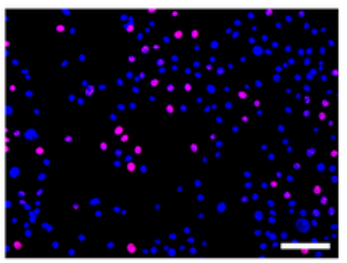

$25 \mu \mathrm{g} / \mathrm{ml}$ Exo

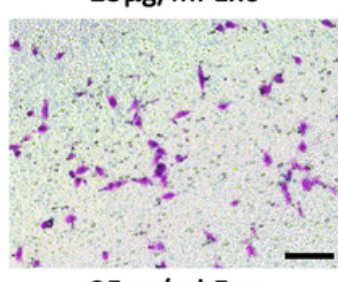

$25 \mu \mathrm{g} / \mathrm{ml}$ Exo
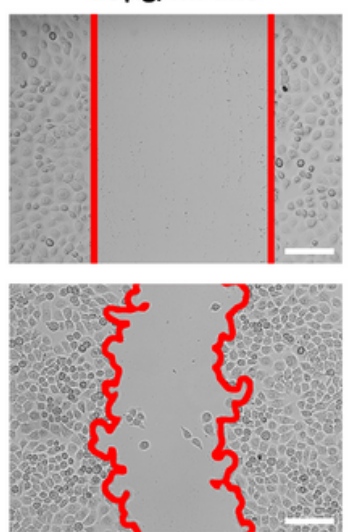

$25 \mu \mathrm{g} / \mathrm{ml}$ Exo

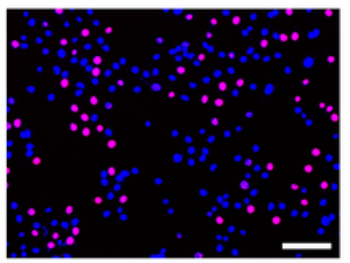

$50 \mu \mathrm{g} / \mathrm{ml}$ Exo

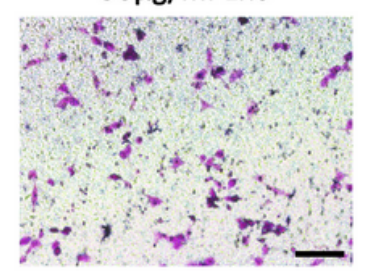

$50 \mu \mathrm{g} / \mathrm{ml}$ Exo
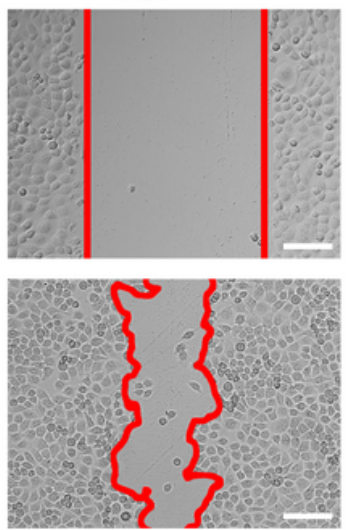

$50 \mu \mathrm{g} / \mathrm{ml}$ Exo

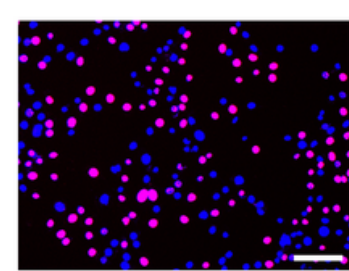

$100 \mu \mathrm{g} / \mathrm{ml}$ Exo

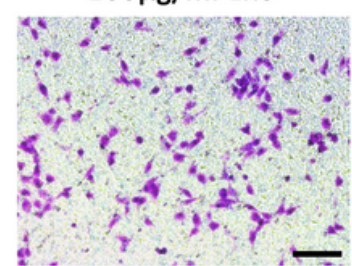

$100 \mu \mathrm{g} / \mathrm{ml}$ Exo
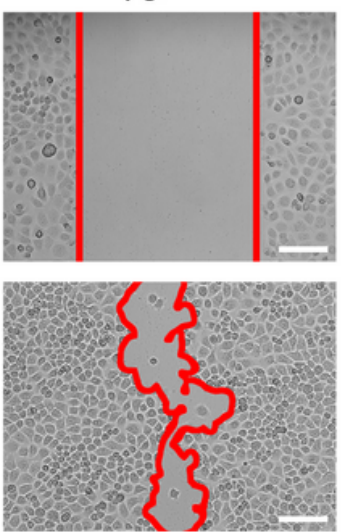

$100 \mu \mathrm{g} / \mathrm{ml}$ Exo
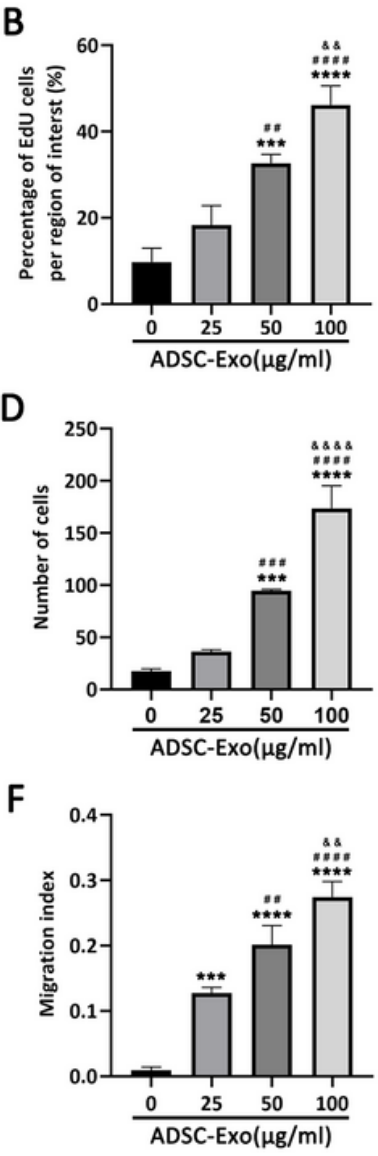

Figure 6

ADSC-Exos significantly promote the proliferation and migration of RPMCs in a dose-dependent manner. A, B Effect of different concentrations of ADSC-Exos on the proliferation of cells by EdU assays. C-F The migration ability of RPMCs treated with ADSC-Exos, measured by transwell and scratch test assays. Bars, $100 \mu \mathrm{m}$. Data are represented as mean \pm SD. *, vs $0 \mu \mathrm{g} / \mathrm{ml}$ group; \#, vs $25 \mu \mathrm{g} / \mathrm{ml}$ group; $\Downarrow$, vs $50 \mu \mathrm{g} / \mathrm{ml}$

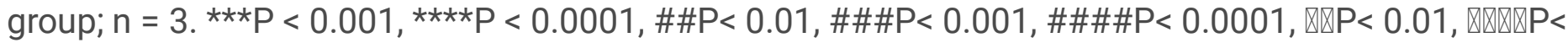
0.0001 
A

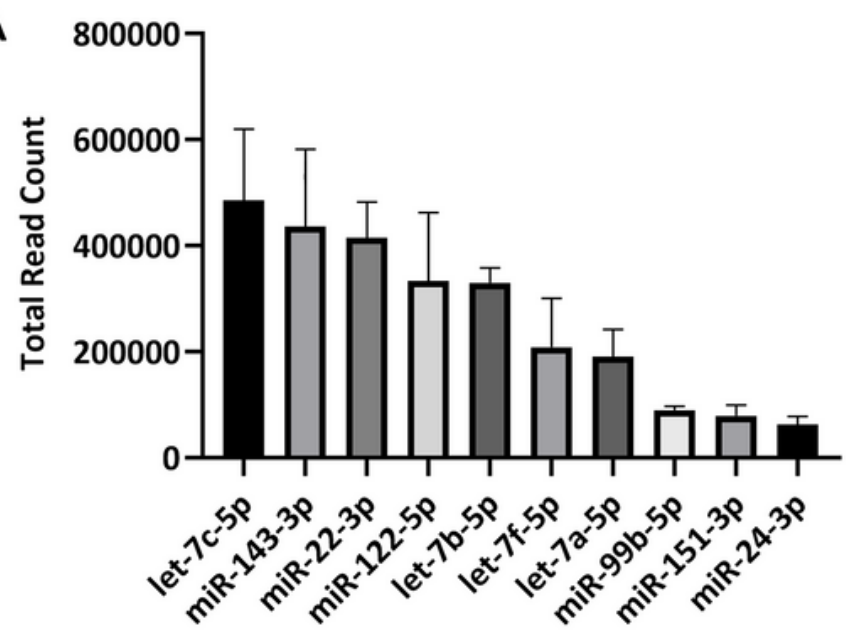

C

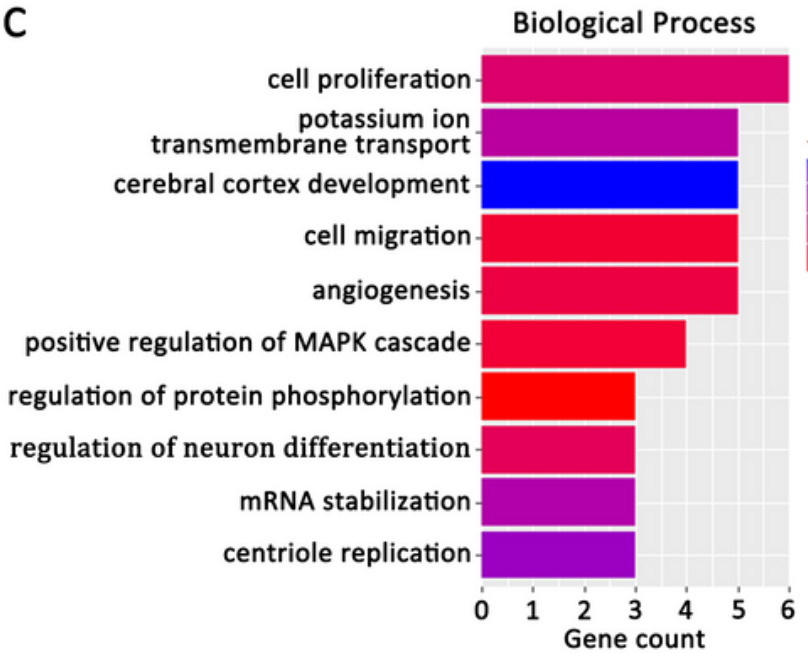

$\mathrm{E}$

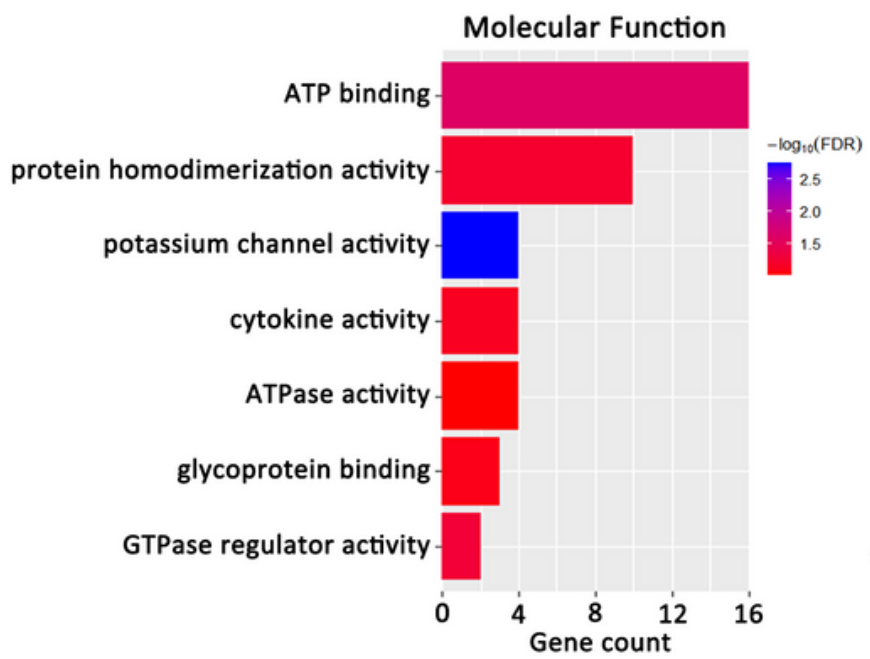

B
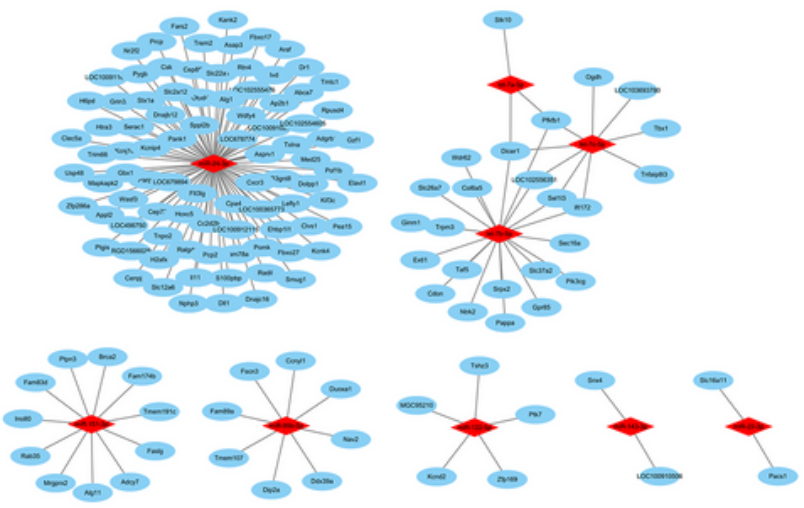

D

Cellular Component

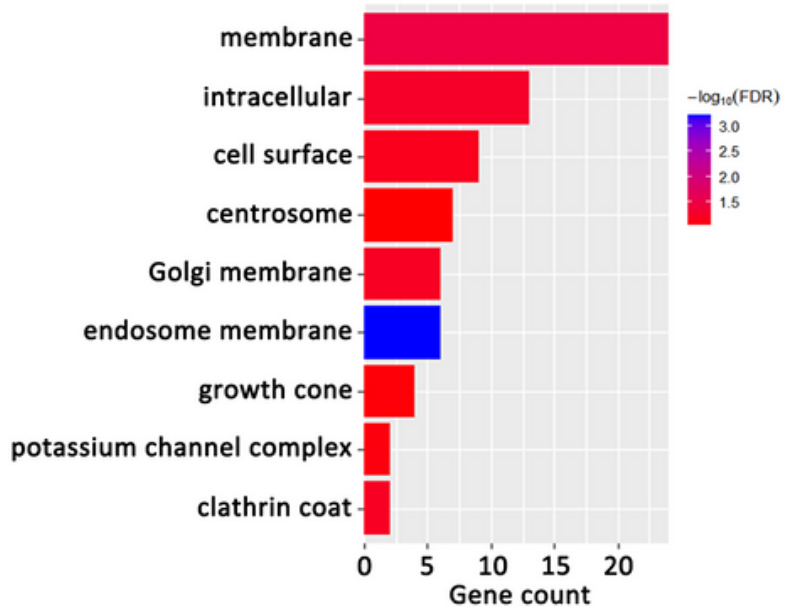

$\mathbf{F}$

KEGG pathways

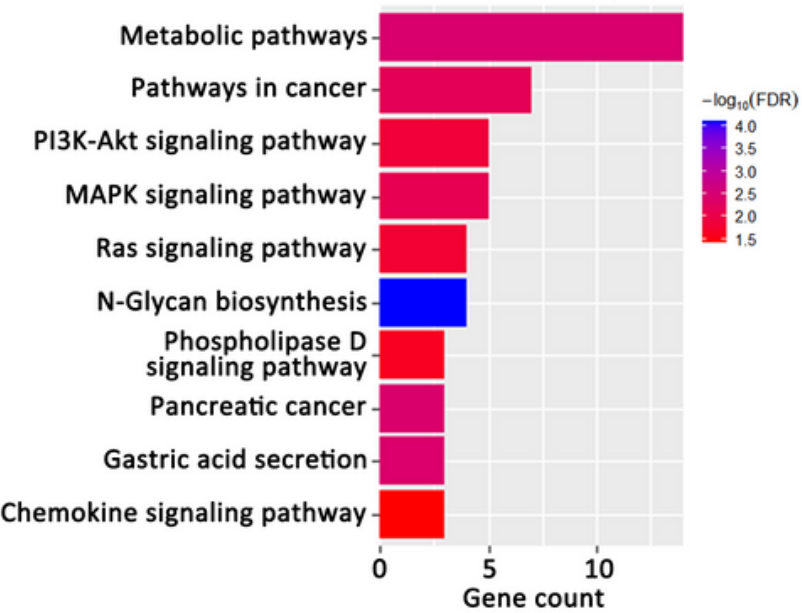

\section{Figure 7}


A

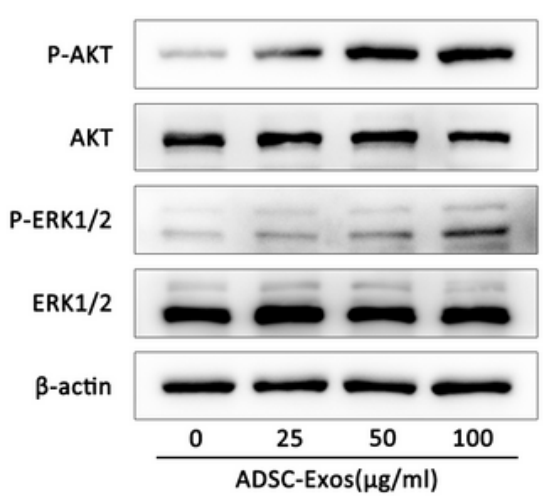

B

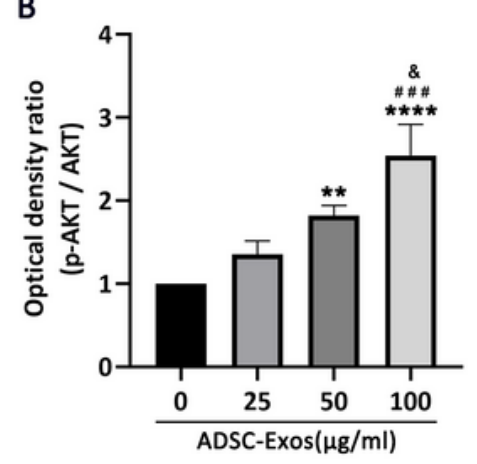

C

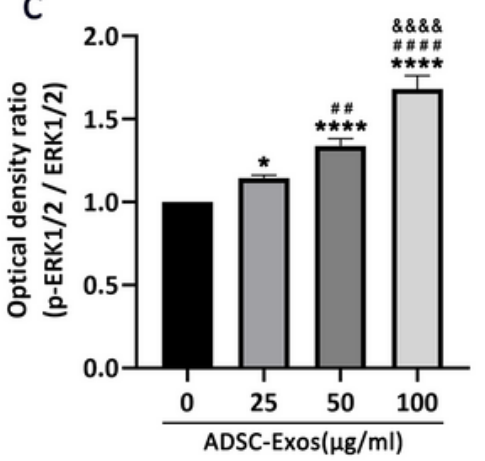

D
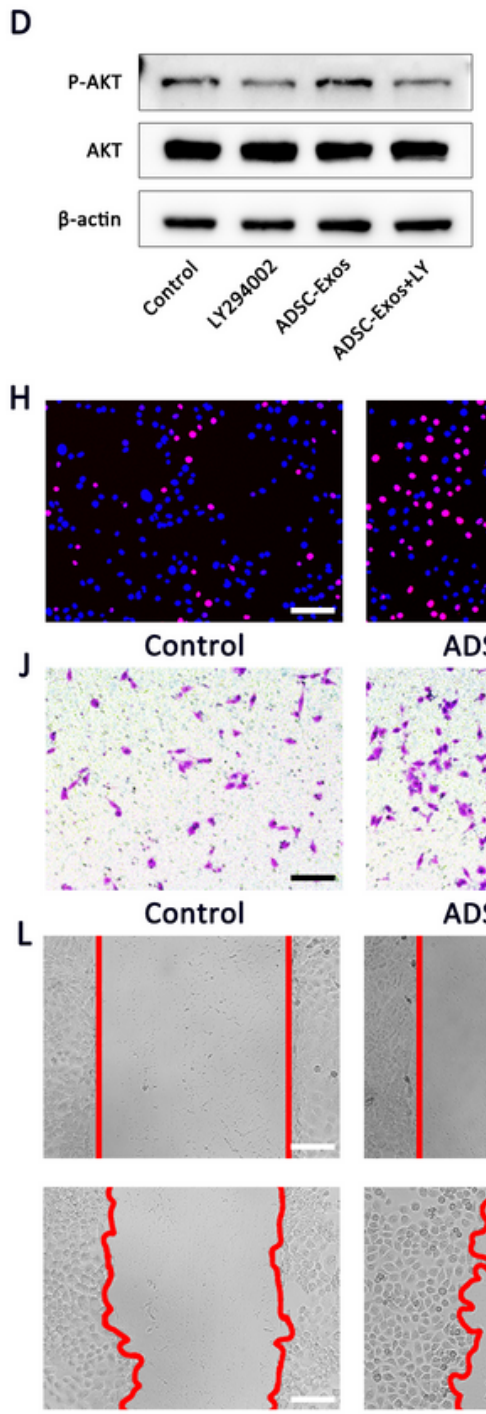

Control
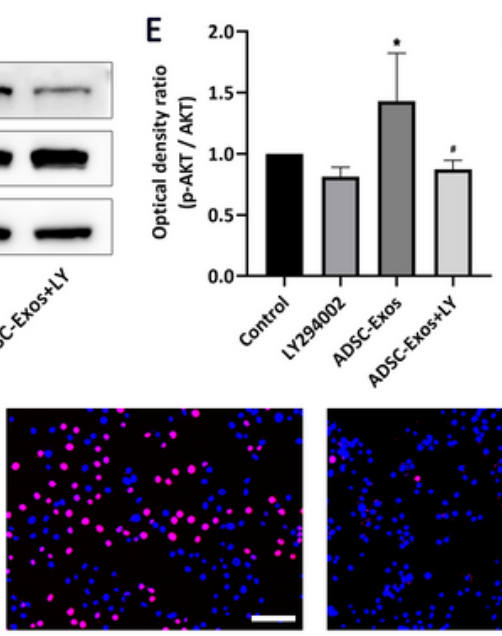

ADSC-Exos
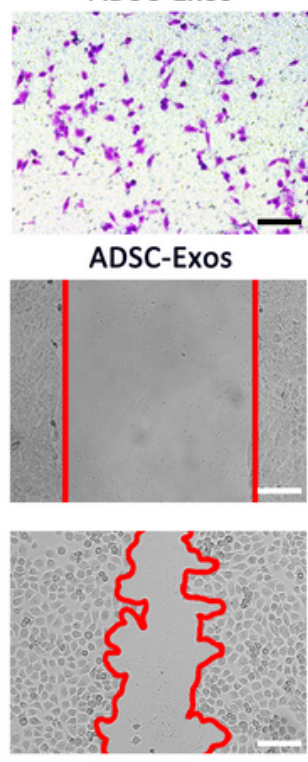

ADSC-Exos

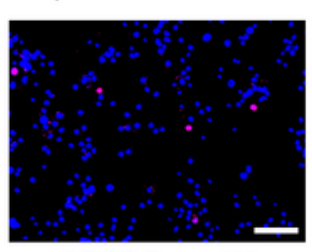

ADSC-Exos+LY
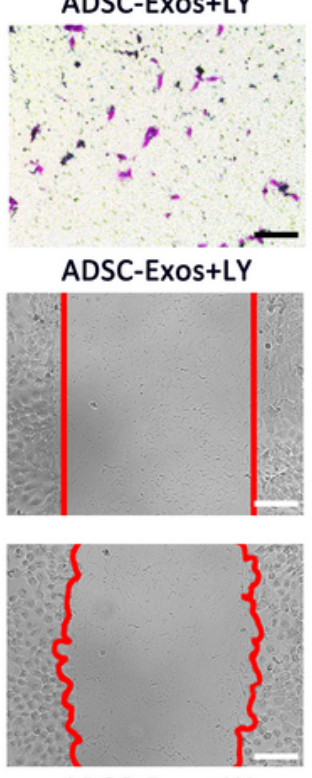

ADSC-Exos+LY

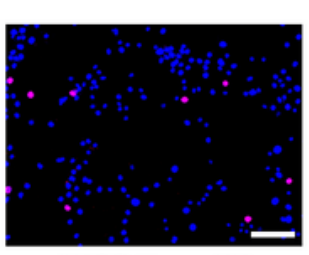

ADSC-Exos+PD

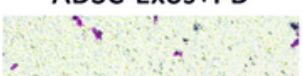

$\mathrm{K}$
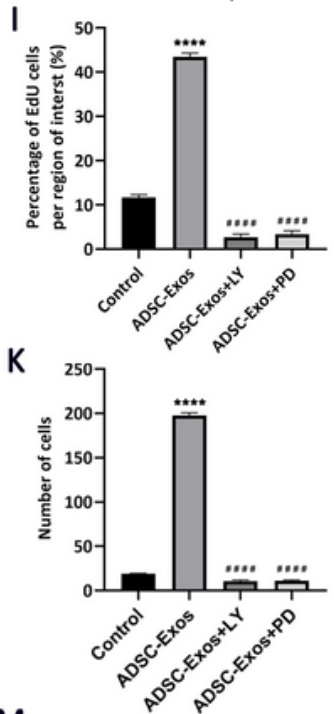

M

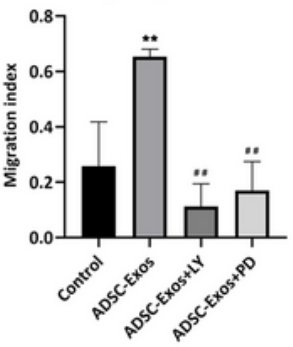

Figure 8

ADSC-Exos promote the proliferation and migration of cells via the PI3K-Akt and MAPK-ERK 1/2 signaling. A-C Western-blot analysis of protein levels of $p$-Akt and $p$-ERK1/2 induced by different concentrations of ADSC-Exos. D-G LY294002 and PD98059 inhibit the activation of PI3K-Akt and MAPKERK1/2 induced by ADSC-Exos, respectively. $\mathrm{H}$, I EdU assay showed that ADSC-Exos-mediated RPMCs proliferation was suppressed by inhibitors LY294002 and PD98059. J-M Transwell and scratch assays 
showed that ADSC-Exos enhanced RPMCs migration at $24 \mathrm{~h}$, but this effect was significantly reduced by inhibitors LY294002 and PD98059. Bars, $100 \mu \mathrm{m}$. Data are represented as mean \pm SD. *, vs $0 \mu \mathrm{g} / \mathrm{ml}$

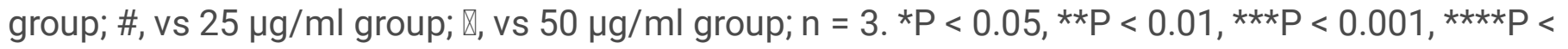

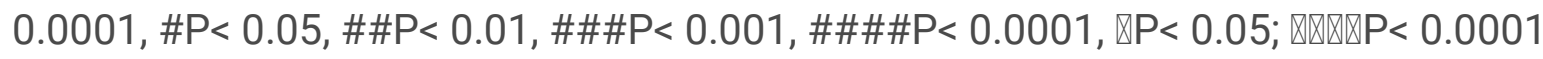

\section{Supplementary Files}

This is a list of supplementary files associated with this preprint. Click to download.

- GA.tif 